PREPARED FOR THE U.S. DEPARTMENT OF ENERGY, UNDER CONTRACT DE-AC02-76CH03073

PPPL-3545

PPPL-3545

UC-70

A Tutorial on Basic Principles of Microwave Reflectometry Applied to Fluctuation Measurements in Fusion Plasmas

by

R. Nazikian, G.J. Kramer, and E. Valeo

February 2001

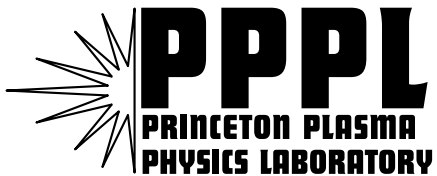

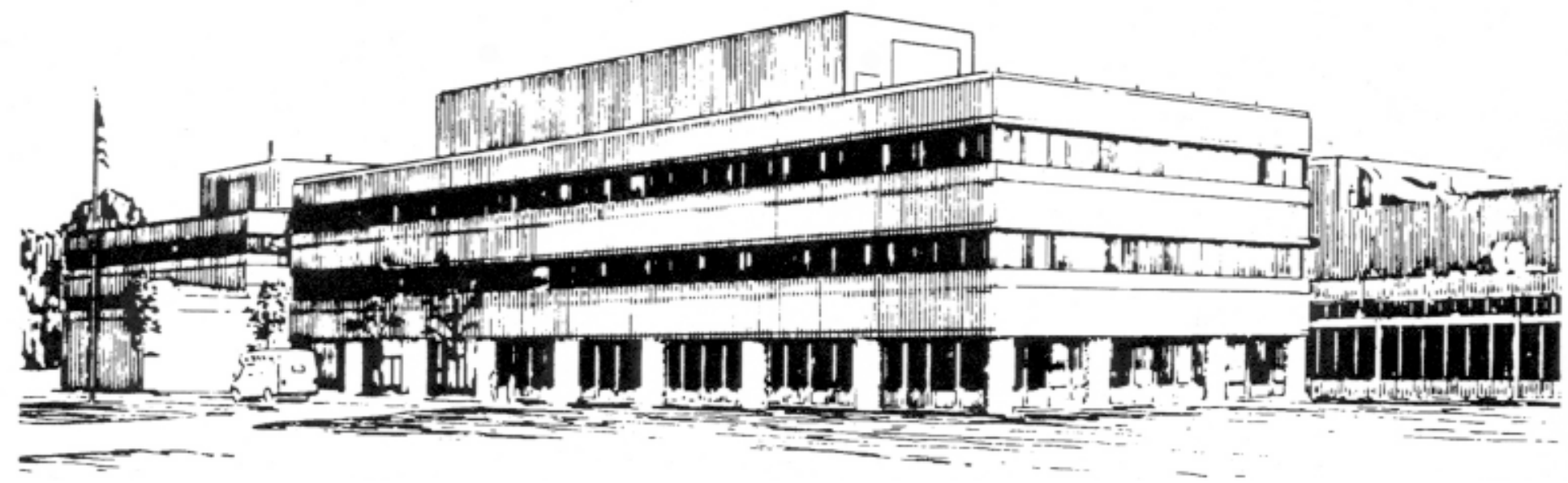

PRINCETON PLASMA PHYSICS LABORATORY PRINCETON UNIVERSITY, PRINCETON, NEW JERSEY 


\section{PPPL Reports Disclaimer}

This report was prepared as an account of work sponsored by an agency of the United States Government. Neither the United States Government nor any agency thereof, nor any of their employees, makes any warranty, express or implied, or assumes any legal liability or responsibility for the accuracy, completeness, or usefulness of any information, apparatus, product, or process disclosed, or represents that its use would not infringe privately owned rights. Reference herein to any specific commercial product, process, or service by trade name, trademark, manufacturer, or otherwise, does not necessarily constitute or imply its endorsement, recommendation, or favoring by the United States Government or any agency thereof. The views and opinions of authors expressed herein do not necessarily state or reflect those of the United States Government or any agency thereof.

\section{Availability}

This report is posted on the U.S. Department of Energy's Princeton Plasma Physics Laboratory Publications and Reports web site in Calendar Year 2001. The home page for PPPL Reports and Publications is: http://www.pppl.gov/pub_report/

DOE and DOE Contractors can obtain copies of this report from:

U.S. Department of Energy

Office of Scientific and Technical Information

DOE Technical Information Services (DTIS)

P.O. Box 62

Oak Ridge, TN 37831

Telephone: (865) 576-8401

Fax: (865) 576-5728

Email: reports@adonis.osti.gov

This report is available to the general public from:

National Technical Information Service

U.S. Department of Commerce

5285 Port Royal Road

Springfield, VA 22161

Telephone: 1-800-553-6847 or

(703) 605-6000

Fax: (703) 321-8547

Internet: http://www.ntis.gov/ordering.htm 


\title{
A Tutorial on the Basic Principles of Microwave Reflectometry Applied to Fluctuation Measurements in Fusion Plasmas
}

\author{
R. Nazikian, G.J. Kramer and E. Valeo \\ Princeton Plasma Physics Laboratory, Princeton NJ 08543-0451
}

\begin{abstract}
Microwave reflectometry is now routinely used for probing the structure of magneto hydrodynamic and turbulent fluctuations in fusion plasmas. Conditions specific to the core of tokamak plasmas, such as small amplitude of density irregularities and the uniformity of the background plasma, have enabled progress in the quantitative interpretation of reflectometer signals. In particular the extent of applicability of the 1-D geometric optics description of the reflected field is investigated by direct comparison to 1-D full wave analysis. Significant advances in laboratory experiments are discussed which are paving the way towards a thorough understanding of this important measurement technique. Data is presented from the Tokamak Fusion Test Reactor [R. Hawryluk, Plasma Physics and Controlled Fusion 33, 1509 (1991)] identifying the validity of the geometric optics description of the scattered field and demonstrating the feasibility of imaging turbulent fluctuations in fusion scale devices.
\end{abstract}




\section{INTRODUCTION}

Achieving the promise of fusion energy requires detailed understanding and eventual control of the underlying collective processes responsible for determining plasma performance. One current area of intense research involves the diagnosis and understanding of the non-linear dynamics of turbulent fluctuations. In recent years great progress has been made in unraveling the complexities of plasma behavior and isolating the relevant non-linear feedback mechanisms which control turbulence and affect transitions to, and maintenance of, various confinement regimes. The quest to understand these instabilities and to challenge leading theoretical models on turbulence and transport has motivated scientists to devise new and ingenious methods to probe fluctuations in the high temperature core of fusion plasmas. In order to challenge theory, experimentalists must devise ingenious new techniques for extracting local fluctuation properties in the plasma with good spatial and temporal resolution.

In this tutorial we describe the development of one of the more exciting and challenging experimental methods used to probe the local properties of waves and instabilities in fusion plasmas. The technique known as microwave reflectometry, a form of microwave radar that uses the plasma as a reflector, has helped to revolutionize our understanding of the relationship between fluctuations and transport by providing high resolution localized measurements of turbulence in fusion plasmas. The method first saw use in probing the height of ionospheric plasmas where it was called ionosonde [1]. These early measurements also indicated the presence of irregularities in the ionosphere indicated by amplitude fading of the reflected waves.[2-4] Out of these observations evolved a branch of statistical optics concerned with the physics of amplitude scintillations (fluctuations) for waves passing through irregular media.[5-9] One of the motivations for making use of waves reflected from plasma is to take advantage of the sensitivity of the probe beam to fluctuations near the turning point or zero in the refractive index. [10-14] The enhanced sensitivity to fluctuations near the turning owes to the decrease in the group velocity as the wave front approaches the cutoff layer, unlike standard scattering techniques that operate well above the plasma cutoff frequency. The advantages of 
localization and the ease of implementation of the diagnostic method has spurred a great deal of experimental and theoretical research into providing a firm foundation for the quantitative interpretation of these measurements. This tutorial will address the underlying physics of reflectometry used in its application to fusion scale experiments and the exciting progress in producing a quantitatively accurate description of the measurements technique.

Since the discovery of the ionosphere in the early part of the $20^{\text {th }}$ century from the reflection of radio waves, the method of reflectometry or Ionosonde (pulsed or frequency swept electromagnetic wave reflection from plasma) has evolved into an indispensable tool for the investigation of the electron height distribution in space and laboratory plasmas.[15-18]. Ionosonde was the first method used to investigate the ionosphere, and it continues to be of great importance today for height determination of the electron density and as a qualitative indicator of the presence of large-scale irregularities. The method is also routinely applied to fusion plasma experiments on many devices and is one of the few techniques which can resolve the sharp density profiles found in plasmas with internal or edge transport barriers.[19-21] Reflectometry can be used both as a measure of density profiles and as a diagnostic of turbulent or coherent fluctuations in the plasma. The method has been extensively investigated theoretically and in controlled laboratory experiments where the correlation properties of the reflected signals can be compared to probe measurements of the density turbulence.[22-27] These studies have greatly increased our quantitative understanding of the technique and its limitations as a probe of turbulent fluctuations.

In order to provide a physically intuitive picture of reflection from plasma irregularities, consider the simplified notion of a wave reflecting from a rippled mirror. A wave reflecting from surface irregularities will experience fluctuations in amplitude, phase and direction of the outgoing waves. These modulations on the reflected waves need to be interpreted in order to extract information on the nature of the surface irregularities. It may be apparent to the reader that an important criterion for the quantitative interpretation of a reflected signal is the degree of uniformity or smoothness of the 
reflecting layer. If the fluctuations are too strong, the reflected wavefront will be overmodulated and a linear relation between the modulation level on the reflected waves and the depth of the surface irregularities will be lost. The advent of advanced confinement regimes with low density fluctuation levels $(<1 \%)$ in the core of the discharge is a major advantage for the quantitative interpretation of reflectometer measurements as low fluctuation levels lead to a simplified linear relation between the scattering amplitude and the level of density fluctuations. A further advance in the method was the use of multiple frequency microwaves in order to sample fluctuations at different radii in the plasma column. The method, known as correlation reflectometry, has evolved into a routine diagnostic of turbulent correlation lengths in major fusion facilities. [28-31] Dramatic examples of fluctuation suppression have been observed with the use of reflectometry in the core of fusion plasmas. [32-34] The radial correlation length of turbulence has also been studied with this method, and the results are consistent with models of enhanced confinement resulting from the radial decorrelation of turbulent eddies.[35-39]

This tutorial covers the basic principles of reflectometry relevant to current experiments in fusion plasmas. In Section II we review the basic principles of wave propagation in inhomogeneous magnetized plasmas. The birefringence of magnetized plasma and the characteristic modes of propagation relevant to fusion plasmas are discussed. Diffraction from a random phase grating is presented in section III as a useful introduction to scattering from density fluctuations near a reflecting layer. The model is particularly instructive in capturing qualitatively all the major features of reflectometer measurements observed in fusion plasmas, although its range of validity must be checked against full wave simulations in realistic geometry. The range of validity of 1-D geometric optics is explored in section IV by comparison to 1-D full wave analysis. It is shown that the 1-D geometric optics description of the scattered field is valid for radial scale lengths down to the width of the interference fringe at the reflecting layer. The validity of the random phase screen approximation in describing the scattering from 2-D irregularities is explored in section V. Here it is shown that the phase screen approximation is indeed a valid description of scattering from turbulent fluctuations in the TFTR device in at least some cases [45]. The validity of the description of the scattering process as diffraction 
from a thin phase changing screen has important implications for future imaging applications of turbulent fluctuations. Conclusions on the present state of the art in reflectometry and future directions in the application of the method are discussed in section VI.

\section{Basics of wave propagation in magnetized plasma}

In order to understand the application of reflectometry to fusion plasmas, it is necessary to go over some important yet straight-forward physical properties of wave propagation in ionized gas. The emphasis will be on making clear how these physical principles impact the design, execution and interpretation of experiments. As such, there will be no attempt at rigor or completeness, there being a vast literature on this topic available to the enthusiastic reader. [40]

A basic fact of magnetized plasma is its birefringence for weak high frequency waves where $\omega>>\omega_{\text {ci }}$ where $\omega$ is the wave frequency and $\omega_{c i}$ is the ion cyclotron frequency. Namely, for a homogeneous plasma (i.e., uniform electron density and magnetic field) there are two characteristic modes of propagation in the plasma of different (orthogonal or counter rotating elliptic) polarization. This is true for any direction of propagation relative to the direction of the magnetic field. There are two very important limiting cases resulting in a much simplified description of the characteristic modes of propagation. The first is for propagation near perpendicular to the direction of the magnetic field (which is most relevant to reflectometer experiments in fusion and equatorial "sounding" experiments in the ionosphere). The second is wave propagation in the direction parallel to the magnetic field, which is most relevant to ionospheric sounding studies in the upper latitudes. Here, sounding is used to denote the study of microwave reflections.

\section{A. The Dispersion relation}


Consider a homogeneous magnetized plasmas, and ignore collisions and ion motion for high frequency waves. The dispersion relation for a plane wave $\mathbf{E}=\mathbf{E}_{0} \exp (i k z-i \omega t)$ propagating at an arbitrary angle $\theta$ to the magnetic field $\mathbf{B}_{0}$ is given by

$$
\left(\frac{c k}{\omega}\right)^{2}=\varepsilon=1-\frac{X}{1-\frac{Y^{2} \sin ^{2} \theta}{2(1-X)} \pm\left[\frac{Y^{4} \sin ^{4} \theta}{4(1-X)^{2}}+Y^{2} \cos ^{2} \theta\right]^{1 / 2}}
$$

where $\varepsilon$ is the plasma permittivity, $X=\omega_{p e}^{2} / \omega^{2}, Y=\omega_{c e} / \omega, \omega_{p e}^{2}=n_{e} e^{2} / \varepsilon_{0} m_{e}$ is the plasma frequency and $\omega_{c e}=|e| B_{0} / m_{e}$ is the electron cyclotron frequency, $m_{e}$ is the electron mass and $n_{e}$ is the electron density. The refractive index is given by $\mu=\sqrt{\varepsilon}$. Turning points, or reflecting layers correspond to locations where $\mu=0$ (infinite wavelength), while resonances correspond to the cases where $\mid \mu \mapsto \infty$ (i.e. where the wavelength goes to zero). Note that cyclotron absorption is ignored which is acceptable as long as the waves do not approach the cyclotron frequency along the path of propagation. In contrast, the real part of the refractive index can be modified by finite electron temperature and this needs to be taken into account. The procedure of inserting an electron mass correction into the dispersion relation $m_{e} \rightarrow m_{e} \sqrt{1+5 T_{e} / M_{e}}$ works very well in reproducing the displacement of the cutoff layer where $M_{e}=511 \mathrm{keV}$ is the electron rest mass and $T_{e}$ is the electron temperature in $\mathrm{keV}$.[41-44]

In the above we have conveniently taken the wave front propagation in the z-direction, and without loss of generality we are free to choose the magnetic field to be orthogonal to the $\mathrm{x}$-direction and hence lie in the z,y plane as shown in Fig. 1. We specify the polarization of the characteristic waves of propagation by $R=E_{x} / E_{y}$ (where $x$ and $\mathrm{y}$ are orthogonal to $\mathrm{z}$ ). Here $\mathrm{R}$ is the ratio of the two components of the electric field orthogonal to the direction of wave propagation. The polarization is then given by 


$$
R=\frac{-i}{Y \cos \theta}\left\{\frac{Y^{2} \sin ^{2} \theta}{2(1-X)} \mp\left(\frac{Y^{4} \sin ^{4} \theta}{4(1-X)^{2}}+Y^{2} \cos ^{2} \theta\right)^{1 / 2}\right\}
$$

Note that in the absence of absorption, the two components of the electric field are always out of phase by $\pi / 2$ radians, so that we may take $|R|$ as directly indicating the degree of ellipticity of the characteristic waves in the medium. The polarization of the two characteristic mode of propagation for a given direction $\theta$ are identical upon interchange of the two axes $\mathrm{x}-\mathrm{y}$, and are counter rotating.

The two cases of most interest correspond to the angles of propagation $\theta=0^{\circ}$ and $90^{\circ}$ to the magnetic field. For $\theta=0^{\circ}\left(180^{\circ}\right)$, corresponding to propagation parallel (anti-parallel) to the field line then $R=+i$ or $-i$. This indicates that the two characteristic modes are circularly polarized and counter rotating with respect to each other, with a dispersion relation $1-X /(1 \pm Y)$ which is sensitive to both the density and the magnetic field strength. This case is of most interest to high latitude sounding experiments in the ionosphere where the magnetic field lines intersect the ground and the plasma density varies with height along the field lines. For $\theta=90^{\circ}$ or $-90^{\circ}$, corresponding to propagation perpendicular to the magnetic field, $R$ gives $E_{x}=0$ or $E_{y}=0$. Note that for $E_{x}=0$, the dispersion relation is independent of the magnetic field strength $(\varepsilon=1-X)$ which corresponds to the Ordinary-mode (O-mode) of propagation where the polarization is parallel to the magnetic field. In addition there is the Extraordinary-mode (X-mode) corresponding to the complementary (perpendicular) polarization state which is both sensitive to the magnetic field and density, $\varepsilon=1-X(1-X) /\left(1-X-Y^{2}\right)$. Here the polarization states are linear in contrast to the general elliptic case for $\theta \neq 90^{\circ}$.

In an inhomogeneous medium, coupling between various modes of propagation can take place, and these couplings are essential for understanding and making use of waves in plasma. Coupling can take place when the wavenumber of two modes with the same frequency coincide. For normal incidence $(\theta= \pm 90$ degrees), coupling can take place 
between oppositely directed waves at the plasma cutoff where $\mu=0$, leading to wave reflection. The use of a reflecting (cutoff) layer (where $k=0$ ) for the investigation of properties of the medium is the essential feature of reflectometry as a diagnostic technique. For propagation perpendicular to field lines (of greatest relevance to fusion applications) the coupling - or turning point - for forward and backward going waves for the O-mode is given by $\omega=\omega_{p e}$ and for the $\mathrm{X}$-mode it is given by $\omega=\left(\omega_{c e}^{2} / 4+\omega_{p e}^{2}\right)^{1 / 2} \pm \omega_{c e} / 2$.

In practice, waves will be launched from an aperture of finite extent and these waves will have a spread of angles relative to the magnetic field. From the experimental point of view it is useful to determine to what extend the limiting case of 90 degree propagation and linearly polarized characteristic waves represents a good approximation for reflectometer experiments in fusion plasmas. In Figure 2, two important cases of interest for wave propagation above and below the electron cyclotron frequency are shown. Plotted is the square of the refractive index $\mu^{2}$ vs $X$ for $Y=0.7\left(\omega_{c e}<\omega<2 \omega_{c e}\right)$, and $Y=1.3$ $\left(\omega<\omega_{c e}\right)$ and for relative angles of propagation to the magnetic field of 90, 80, 70 and 60 degrees. For any value of $\mathrm{X}$ there are two possible modes, one corresponding to the $\mathrm{O}$ mode (with polarization parallel to the magnetic field for normal incidence) and the Xmode (polarization orthogonal to the magnetic field for normal incidence). Also shown is the degree of ellipticity $|R|$ for these two cases in Figure 3. For inclinations of much less than 10 degrees to the normal, (typical of high gain antennas in fusion experiments) the refractive index is very close to that of normal incidence and the degree of ellipticity is small. Note from Fig. 2 that the zeros of the refractive index are independent of the angle of inclination to the magnetic field, while the location of the upper hybrid resonance in the X-mode branch (where the refractive index diverges) depends on angle. For all but small angles to the magnetic field the degree of ellipticity of the characteristic modes is considerable, and can represent $>10 \%$ of the amplitude of the mode. Thus, a linearly polarized wave launched from an antenna at 10 degrees off perpendicular to the magnetic field will couple to both $\mathrm{O}$ and $\mathrm{X}$ modes of propagation and possibly lead to mixed signals. For $Y=0.7$, the so called upper Right hand $(\mathrm{RX})$ cutoff is encountered at a lower 
density than the O-mode cutoff and the upper hybrid resonance occurs at higher density. The cutoff layer in the X-mode at even higher density is called the left hand X-mode cutoff (LX) which is not accessible from vacuum due to the presence of the upper hybrid resonance. For $Y=1.3$, corresponding to propagation below the cyclotron frequency, the O-mode cutoff is encountered at a lower density than the LX cutoff. The practical application of these cutoffs will be discussed in the next section.

\section{B. Wave propagation in a spatially varying plasma}

How can we use the information on local dispersion above to design a real experiment? What do we launch and what do we measure? First note that in a slowly varying plasma, where the medium changes little in one wavelength of the probe beam and where the refractive indicies of the modes are distinct, then the characteristic modes essentially do not couple. Appreciable coupling can and does take place between the characteristic modes when the magnetic pitch angle in the plasma varies sufficiently rapidly. This coupling is the basis for proposed methods to measure the magnetic field line pitch angle in fusion plasmas. $[15,17]$ However, for the most part we can consider the characteristic modes in Tokamaks as being independent of each other.

Figure 4 shows the cutoff layers for $\theta=90^{\circ}$ propagation as a function of major radius for the case of a low density Ohmic plasma on TFTR [45] with the following plasma parameters: $B_{T}=4.5 \mathrm{~T}, \mathrm{n}_{\mathrm{e}}(0)=2 \times 10^{13} \mathrm{~cm}^{-3}, \mathrm{I}_{\mathrm{p}}=1.1 \mathrm{MA}$, major radius $\mathrm{R}=2.6 \mathrm{~m}$. Note that the case of $\mathrm{Y}>1$ and $\mathrm{Y}<1$ are shown. To access the RX cutoff the waves need to propagate between the fundamental and second harmonic electron cyclotron frequency. Also, the Omode and LX cutoff layers are accessible for $\mathrm{Y}>1$. The RX, $\mathrm{O}$ and LX cutoffs are all accessible from the low magnetic field side (large major radius side) of the plasma. However, the RX cutoff is not accessible from the high magnetic field side of the plasma due to the presence of the upper hybrid resonance (shown between the electron cyclotron and RX cutoff). The use of high field side propagation to the LX cutoff was proposed as a means of measuring the core density in plasmas with flat density profiles and was tested on the Doublet III-D device (DIII-D). [46] 
The second piece of information needed to address propagation in inhomogeneous media is based on the simple principle that the only component of the wavelength which changes is that which lies in the direction in which the medium varies. For simplicity, assuming a vertically stratified density in $z$ and uniformity in $x$, then the wavelength in the $\mathrm{x}$-direction will remain constant. Thus, only the z-component of the wavelength varies as the wave propagates. Taking $k_{x}$ as the unchanged component of the incident wavevector in the $\mathrm{x}$-direction, then the wave vector depends on height according to $\mathbf{k}=k_{x} \hat{\mathbf{x}}+k_{z} \hat{\mathbf{z}}$ where $k_{z}^{2}=k_{0}^{2} \mu^{2}\left(z, k_{x} / k_{z}\right)-k_{x}^{2}, k_{0}$ is the incident wave number in free space, $\hat{\mathbf{x}}, \hat{\mathbf{z}}$ are unit normals.

In order to determine the direction of propagation of a signal, we can consider instead the propagation of a localized disturbance such as a beat wave generated by the product of two waves with similar frequency. Both waves must satisfy the local dispersion relation for the characteristic mode of propagation. Given frequencies $\omega_{1}, \omega_{2}$ and wavevectors $k_{1}, k_{2}$ the beat wave propagates with frequency $\Omega=\omega_{1}-\omega_{2}$ and wavenumber $\mathbf{K}=\mathbf{k}_{1}-\mathbf{k}_{2}$. Thus, as $\Omega \rightarrow 0$ the velocity of the beat wave is given approximately by $\mathbf{v}_{g}=\partial \omega / \partial \mathbf{k}$ which is the usual definition of the group velocity of a wave packet typically represented by a ray. A superposition of such beat waves can create a single pulse of information represented by the trajectory of a ray $d \mathbf{r} / d t=\mathbf{v}_{g}$. Given the trajectory of the wave, the phase change along that ray depends of the alignment of the wave fronts to the direction of propagation. The net phase change along a ray is then given by

$$
\phi(\mathbf{r})=\int^{\mathbf{r}} \mathbf{k}\left(\mathbf{x}^{\prime}\right) \cdot d \mathbf{x}^{\prime}
$$

where the integration takes place over the trajectory of the ray and the total time delay of the pulse along the trajectory is given by 


$$
\tau_{g}=\int \frac{d l}{\mathrm{v}_{g}}
$$

where the integral is along the trajectory of the ray. In Figure 5 we show the trajectory of the four $\mathrm{X}$-mode rays used in Figure 2 for $Y=0.7$ corresponding to propagation above the cyclotron frequency and a linear variation of plasma density $(X)$ in the vertical direction. Two sets of ray trajectories are shown corresponding to tilt angles in the direction of the magnetic field (equivalent to the toroidal direction in a tokamak) and tilt angles perpendicular to the magnetic field (corresponding to the poloidal spread of rays in a tokamak). For either set of curves, the lateral drift of the ray can be quite severe as the angle of inclination increases, and its precise turning point and trajectory may differ considerably from waves at normal incidence. The spread in the turning point location will be minimal for high gain antennas with narrow beam angles $\left(\theta \approx 90^{\circ}\right)$. In fusion plasmas, reflectometry only works if you can get the reflection back into the receiver and the receiver is usually located close to the transmitting antenna. The condition for obtaining a reflection back to the transmitter location is most easily satisfied on the plasma midplane, so that reflectometer measurements usually probe this region of the plasma.

We now have in place a description of wave propagation in plasma which allows the design of experiments and interpretation of some measurements. Experimentally, we want to send a probe beam of well defined characteristics (direction, polarization and frequency) into the plasma and we want to interrogate the beam on its way out to learn about the properties of the medium. These variables can be the polarization, ray trajectory, ray displacement, group delay and phase change. In practice only waves returning to the launch site are measured so that we may assume that the ray paths are very nearly normal to the reflecting layer on the plasma midplane. In such a case, the rays propagate in a straight line along the plasma midplane and the polarization is linear. For rays of normal incidence, the phase of the reflected wave is given by 


$$
\phi=2 k_{0} \int_{0}^{z_{c}} \sqrt{\varepsilon} d z+\pi / 2
$$

where $z_{c}$ is the location of the cutoff layer and $\varepsilon=(c k / \omega)^{2}$. This expression, or the one relating to the group delay, forms the basis for electron density vs height determination in ionospheric and fusion plasmas. It is also the basis for the phase screen model and for the geometric optics description of reflections from random irregularities.

As for fluctuation studies, it is far from clear that Eq. 5 can characterize the properties of waves reflected from density irregularities in a fusion plasma. The experimental observation of large amplitude fluctuations, and large secular drifts in the measured phase suggest that multi-dimensional effects are required to model the experimental data. A schematic diagram of scattering from random eddies and the receiver geometry is shown in Figure 6a. This figure illustrates the effect of a wavefront passing through irregularities and scattering from a rippled cutoff layer. The waves propagating away from the cutoff region and scatter in various directions. The net result is a superposition of waves reaching the receiver from different directions and possessing different amplitudes and phases. The sum of these waves leads to amplitude and phase fluctuations known in the ionospheric community as scintillation phenomena or fading. [2-9] A method used to probe the structure of irregularities in fusion plasmas is known as correlation reflectometry which is illustrated in Figure 6b. $[19,28]$ The method makes use of the difference in the cutoff layer position of waves launched at different frequencies and is used to correlate signals received simultaneously from two different cutoff layers. The separation of the layers is varied by changing the frequency of one of the microwave sources. Analysis is performed using standard statistical measures for the complex amplitude of the detected waves to determine the scattering amplitude and the correlation properties of the received waves.[20-25] The essential issue of reflectometry is how to relate these measured signal correlations to the correlation properties of the fluctuations. A very useful approximation to the scattered field in the limit of long fluctuation wavelengths can be obtained using the ray description, as will be shown. It is a remarkable fact that the combination of 1-D geometric optics with diffraction theory from 
random rough surfaces captures many of the properties of reflectometer signals observed in experiment, at least qualitatively. In the following section we provide a description of the correlation properties of reflectometer measurements using the approximation that the entire scattering process can be represented by a thin phase changing mirror.

\section{Diffraction from a phase changing screen in the 1-D geometric optics limit}

The use of the 1-D phase equation for reflectometry (Eq. 5) has proven sufficient to extract information on the bulk plasma profiles. If we assume the expression holds for large and small scale fluctuations in the plasma then a theory of diffraction can be developed which qualitatively reproduces many of the features of the experimental data and thus serves as a starting point for more general models.[47,24,6]

The key feature of all phase screen models - which makes the physics of reflectometry intuitively clear and analytically tractable - is the separation of the interaction region from the propagation region. Namely, the part of the problem which represents the plasma density fluctuations is separated from the part of the problem which represents wave propagation and diffraction. It is clear from such a separation of interaction and propagation processes that this treatment has no basis for application to actual experiments except by direct confirmation using more sophisticated full wave analysis or by identifying special properties of the experimental data (section V). However, it is precisely the confirmation of such a simplified picture from early (rudimentary slab model) full wave simulations that justified its quantitative application to real experiments on TFTR. It is important to recall that the model itself had its origins in attempts to develop a description of ionospheric reflectometer measurements, with extensive work performed in the late 1950s and early 1960s. This early ionospheric work provides a rather comprehensive treatment of the subject which forms the starting point for modern developments.

\section{A. The phase screen model}


The essence of the phase screen model is that the entire propagation region inside the plasma can be compressed into a thin screen. The thin screen has the property that the depth of the irregularities on the surface are directly related to the 1-D phase integral of geometric optics (Eq. 5). The waves then propagate away from the screen as if in free space, and the screen is placed at a distance to the observer (the effective distance) at which the scattered field appears to originate. As a first step, the phase of geometric optics can be linearized for small density perturbations such that the perturbed phase is given by

$$
\delta \phi=k_{0} \int_{c}^{z_{c}} \frac{\delta \varepsilon}{\sqrt{\bar{\varepsilon}}} d z
$$

where $\bar{\varepsilon}$ is the permittivity of the unperturbed plasma and $\delta \varepsilon=\delta n \cdot \partial \bar{\varepsilon} / \partial n$. Here propagation is taken in the z-direction as indicated in Figure 6, and perturbations along the path of propagation will be distributed in the $\mathrm{x}-\mathrm{z}$ plane. This expression shows how the dominant contribution to the perturbed phase can occur very close to the reflecting layer due to the factor $1 / \sqrt{\bar{\varepsilon}}$ in the integrand. For the O-mode and for a density perturbation of the form $\delta n_{e} \cos \left[K_{z}\left(z-z_{c}\right)\right]$ where $z_{c}$ is the location of the cutoff layer, we obtain the particularly simple expression for the perturbed phase

$$
\begin{array}{ll}
\delta \phi \approx 2\left(k_{0} L_{n}\right) \frac{\delta n_{e}}{n_{e}}, & K_{z} L_{n}<1 \\
\delta \phi \approx 2 k_{0}\left(\frac{L_{n}}{K_{z}}\right)^{1 / 2} \frac{\delta n_{e}}{n_{e}} & K_{z} L_{n}>1
\end{array}
$$

where $L_{n}$ is the density scale length at the cutoff layer, $K_{z}$ is the wavenumber of the density perturbation in the direction of wave propagation. [13] A similar expression can be derived for the $\mathrm{X}$-mode taking into account the density and magnetic field scale lengths. For edge measurements in a tokamak, taking $k_{r} L_{n} \sim 1, k_{r}<k_{0} \approx 1 \mathrm{~cm}^{-1}$ and 
$\delta n / n \approx 0.5$ we have $\delta \phi \approx 1$. A key point here is that there is only a weak dependence of the phase perturbation $\delta \phi$ to the radial scale length of the fluctuations in the plasma, particularly for small $L_{\mathrm{n}}$ relevant to edge plasma measurements.

We now introduce the concept of a thin phase screen (equivalently a phase changing mirror) where the magnitude of the phase change for each point on the screen is given by the integral in Eq. 5. A schematic diagram of such a phase screen is shown in Figure 7a for a sinusoidal perturbation. Consider a sinusoidal phase perturbation of the form $a \cdot \sin \left(K_{x} x-\Omega t\right)$ illuminated by a plane wave $E_{0}=A \exp i\left(k_{0} z-\omega_{0} t\right)$ where $K_{x}$ is the wavenumber of the phase screen and $\Omega$ is its frequency. For a pure phase modulated beam the total field $E$ at the output of the thin screen is given by $E=E_{0} \exp i\left[a \cdot \sin \left(K_{x} x-\Omega t\right)\right]$ which decomposes into multiple sidebands or diffraction orders symmetrically distributed about the direction of the incoming wave as shown in Fig. 7a. Expanding the perturbed field into a Bessel series and noting momentum conservation $\left(k_{\text {scatter }}\left|=k_{0}\right|\right)$ we obtain at an arbitrary distance from the screen

$$
E=A \sum_{m=-\infty}^{\infty} J_{m}(a) \exp i\left[m K_{x} x+\sqrt{k_{0}^{2}-m^{2} K_{x}^{2}}\left(z-z_{c}\right)+\left(\omega_{0}+m \Omega\right) t\right]
$$

where $J_{m}(a)$ is the $\mathrm{m}^{\text {th }}$ order Bessel function and $z-z_{c}$ is the distance from the phase screen. Note that back scattering (the diffraction components propagating in the opposite direction to the incoming wave) are not considered in this expansion. For $a<<1$, only the $\mathrm{m}=0,-1,1$ components of the scattered field are significant. In this case Eq. 8 can be rewritten as

$$
E=A\left\{1+2 i \delta \phi \sin \left(K_{x} x-\Omega t\right) \cdot \exp \left(i \Delta K_{z}\left(z-z_{c}\right)\right)\right\}
$$

where $\Delta K_{z}=\sqrt{k_{0}^{2}-K_{x}^{2}}-k_{0} \approx-K_{x}^{2} / 2 k_{0}$. This simple expression in Eq. 9 goes to the heart of the phase screen model and it also leads directly to a simple test for its validity. At the screen location, the signal is purely phase modulated. From Eq. 9, setting $z=z_{c}$ gives 
$E=A\left\{1+2 i \delta \phi \sin \left(K_{x} x-\Omega t\right)\right\}$ indicating that the perturbation is phase like. Figure $7 \mathrm{~b}$ illustrates this point by showing that the scattered field at the phase screen location $\tilde{E}_{z=z_{c}}$ is orthogonal in complex amplitude to the incident field $E_{0}$, producing only a phase change. However, when $\Delta K_{z}\left(z-z_{c}\right) \sim$ we see that a significant component of the perturbed field has now rotated in the complex phase plane with respect to the incident wave, and this now represents a significant amplitude modulation of the signal. For $\Delta K_{z}\left(z-z_{c}\right)=\pi / 2$, Eq. 9 becomes $E=A\left\{1-2 \delta \phi \sin \left(K_{x} x-\Omega t\right)\right\}$ which represents a pure amplitude modulation. As the distance increases the signal will rotate from a pure phase to a pure amplitude modulation and back as illustrated in Figure $7 \mathrm{~b}$.

This very simple concept has a very powerful application. At the location of the phase screen all the modes are coincident and all produce a pure phase modulation. As the distance from the screen increases, the vectors $\tilde{E}$ associated with the higher wavenumber components of the screen begin to rotate more rapidly with distance than the longer wavelength components, producing a spread of rotations and a combination of amplitude and phase fluctuations. At a far enough distance from the screen, the complex amplitudes of all the scattered waves will appear totally randomized with respect to each other, leading to a Gaussian-like distribution where the real and imaginary components of the field appear independent, corresponding to the familiar Rice distribution. However, if we can identify the rotation of the scattered waves in the complex plane due to the effect of propagation, and if that rotation for each scattered component of the field corresponds to the same effective distance, then the validity of the phase screen model can be determined from the experimental data.

\section{B. Spatial moments of the complex amplitude}

In the previous section we discussed properties of the amplitude and phase of the scattered field, and the transition from a phase modulated wavefront to a random complex behavior producing a Rice distribution far from the screen. In this section we discuss moments (correlations) of the signal in order to determine a quantitative means for 
measuring the correlation properties of the fluctuations and the rotation angle of the scattered components as illustrated in Figure 7.

Correlation properties of the scattered field are used to infer statistical properties of the medium, so it is essential to understand how the correlation behavior of the scattered field varies with propagation distance. Unlike a point measurement, reflectometry involves the remote detection of waves which propagated some distance from the point of interest.

The complex amplitude of the scattered field possesses certain invariant (or near invariant) qualities which makes its behavior a weak function of distance from the screen and can therefore be used to determine the correlation properties of the medium. Indeed, it is the propagation invariance of certain moments of the complex amplitude that makes reflectometry a practical tool for diagnosing irregularities in the plasma. Assuming a phase screen with random Gaussian distributed irregularities and rms phase fluctuations $\sigma_{\phi}=\left\langle\delta \phi^{2}\right\rangle^{1 / 2}$ (where $\delta \phi$ is the perturbed phase in Eq. 6) then for an incident plane wave of unit amplitude the mean of the diffracted field is given by [24]

$$
\left\langle E \mathbf{\rangle}=\exp \left(-\sigma_{\phi}^{2} / 2\right)\right.
$$

Experimentally this corresponds to the unscattered component of the signal appearing at the center frequency of the mixed signal output. Note that as the magnitude of fluctuations $\delta \phi$ increases the coherent reflection decreases, presenting a practical upper limit to the fluctuation level which can be inferred from the measurement of the complex amplitude (typically $\sigma_{\phi} \leq 1$ ). The density fluctuation level for which a coherent or specular reflection cannot be resolved varies dramatically from $<1 \%$ in the core plasma up to 10 $20 \%$ at the edge, depending on the density scale length and the wavelength of the probe beam.

Consider the correlation between any two points on two partially correlated phase screens. This is identical to considering the correlation of reflected waves from two 
radially separated cutoff layers. Given the correlation between two phase screens $\left\{\delta \phi_{1} \delta \phi_{2}\right\rangle=\sigma_{\phi}^{2} \gamma_{\phi}\left(\mathbf{r}_{1}-\mathbf{r}_{2}\right)$ with displacement $\mathbf{r}_{\mathbf{1}}-\mathbf{r}_{\mathbf{2}}$ where the normalized correlation is $\gamma_{\phi}(0)=1$. We then have for the correlation of the scattered field

$$
\left\{E_{1} E_{2}^{*}\right\rangle=\exp \left[-\sigma_{\phi}^{2}\left(1-\gamma_{\phi}\left(\mathbf{r}_{1}-\mathbf{r}_{2}\right)\right)\right]
$$

Note that Eq. 11 is propagation invariant for a homogeneous random screen, so that the correlation function is identical on any plane of detection beyond the scatterer. This represents a convenient property of the radiated field which simplifies analysis. For small fluctuation levels $\left(\sigma_{\phi}<1\right)$ the correlation of the complex amplitude is identical to the correlation between the two phase screens. As long as there is a significant coherent reflection (<E $>\sim 0.5-1.0)$, the correlation coefficient of the measured electric field $\left\{E_{1} E_{2}^{*}\right\rangle$ together with the coherent reflection $\langle E\rangle$ can be used to infer the correlation coefficient of the phase of geometric optics $\gamma_{\phi}$ between the two phase screens. However, Eq. 11 cannot be used to confirm the validity of the phase screen model for imaging applications. In order to do this, a further property of the scattered field needs to be considered based on sideband correlations. [48,49]

\section{Sideband correlation and the phase screen model}

The correlation between symmetric diffraction orders in the field scattered from a random phase screen is given by [48]

$$
\left\{E\left(K_{x}, \omega_{0}+\Omega z\right) E\left(-K_{x}, \omega_{0}-\Omega ; z\right)\right\rangle=e^{-i 2 \Delta K_{z} z} \iint d x d t e^{-i(K x-\Omega t)} e^{-\sigma_{\varphi}^{2}\left(1+\gamma_{\phi}\right)}
$$

where the sidebands of the scattered field with components $\pm K_{x}$ are illustrated in Figure 7a. The importance of this expression is that for sufficiently weak fluctuations $\left(\sigma_{\phi}^{2}<<1\right)$ and there is a phase $\exp \left(2 i \Delta K_{z} z\right)$ which can be measured and corresponds to twice the 
rotation angle of the scattered component of the field in Figure $7 \mathrm{~b}$. This phase term in the sideband correlation indicates the distance of the phase screen to the point of measurement, while the magnitude of the correlation indicates the symmetry of the scattered spectrum. If the sidebands are highly and the phase varies as the square of the wavenumber $K_{x}$ then the scatterer can be considered a this phase changing screen. In addition to determining the validity of the phase screen approximation, a numerical transformation (by undoing the phase $\operatorname{shift} \exp \left(i \Delta K_{z} z\right)$ for each Fourier component of the scattered field) will allow for the numerical imaging of the fluctuations without the use of imaging optics. If the effect of phase curvature can be undone, by applying an appropriate phase correction, then this scheme is equivalent to numerically imaging the screen by the removal of phase dispersion due to propagation. Later we will show data taken on TFTR which yields a high degree of sideband correlation and is consistent with the phase screen approximation.

\section{Full wave analysis in one dimension}

The preceding discussion established the relation between the approximation of geometric optics, correlation properties of the scattered field and its relation to the density correlations in the medium. However, there are some very fundamental issues concerning this approach which need to be resolved with full wave analysis. Namely, we need to confirm under what conditions the geometric optics approximation adopted in the preceding sections represents a valid description of the scattering process from an extended plasma source. In order to do this, detailed comparison is required between the geometric optics solutions and the solutions of the 1-D full wave equation incorporating a spectrum of density fluctuations and a range of correlation lengths.

\section{A. Description of the 1-D Wave Solver}

In comparing the solutions of geometric optics to the full wave analysis in 1-D we solve for the complex amplitude of the scalar wave equation 
$\left(\frac{\partial^{2}}{\partial x^{2}}+k_{0}^{2} \varepsilon\right) E=0$

where $\varepsilon$ is the scalar approximation to the plasma permittivity for paraxial waves with a zero (cutoff) somewhere in the integration region and the wave field

$E=\exp \left(i k_{0} x\right)+U \exp \left(-i k_{0} x\right)$

represents the the sum of the inward $\left[\exp \left(i k_{0} x\right)\right]$ and outward $\left(U \exp \left(-i k_{0} x\right)\right)$ propagating component in vacuum. For a real permittivity, only the phase of the outward solution is modified so that $U_{\omega}=\exp \left(i \varphi_{\omega}\right)$ where $\omega$ is the wave frequency. The wave amplitude is taken to be zero on the opposite edge of the integration domain, and the wave equation is solved using a tri-diagonal implicit method. The permittivity is taken for either $\mathrm{O}$ or $\mathrm{X}$ mode propagation and the coupling between modes due to magnetic field line pitch angle variation is ignored. Although the expression for the permittivity contains the upper hybrid resonance for the upper X-mode, in practice there are many wavelengths between the cutoff and the resonance, making tunneling exponentially weak.

Figure 4 shows an example of the radial variation of cutoff frequencies for a typical ohmic TFTR discharge. We compute the solution to the full wave equation for this discharge in order to analyse the localization properties of reflectometry to fluctuations near the cutoff. Figure 8 illustrates the sensitivity of the reflectometer response to fluctuations near the cutoff layer for a model perturbation of the form $\tilde{n}_{e}=a \cdot \exp \left(-\left(z-z_{0}\right)^{2} / W^{2}\right) \cos \left(K\left(z-z_{0}\right)\right)$. In fig. 8a, the solution of the wave equation is plotted as a function of the major radius for X-mode propagation at $115 \mathrm{GHz}$. The fluctuation level of the density perturbation is taken as $0.1 \%$ of the electron density at the cutoff. Figure 8b shows $\tilde{\varphi}=\varphi\left(z_{0}\right)-\varphi(-\infty)$ where $\varphi\left(z_{0}\right)$ is the phase of $U$ for $\tilde{n}_{e}$ centered at $z_{0}$. As the packet is moved through the reflecting layer (i.e., $z_{0}$ is scanned) the response $\varphi\left(z_{0}\right)$ appears to be highly localized to the perturbtion centered near the cutoff layer. The first case (7b) corresponds to a packet width of $0.5 W_{\text {Airy }}$ where $W_{\text {Airy }} \approx 0.5 L_{\varepsilon}^{1 / 3} \lambda_{0}^{2 / 3}$ is approximately the width of the last fringe of the full wave solution 
at the cutoff layer and $L_{\varepsilon}$ is the permittivity scale length. This result indicates that the scale length of the irregularity can be at least as small as $W_{\text {Airy }}$ without significant degradation of sensitivity or localization. At larger scales, for perturbations much larger than $W_{\text {Airy }}$, the phase response $\varphi\left(z_{0}\right)$ remains localized to density perturbations near the reflecting layer as shown in Figure 8c. These examples illustrate that for both short and long scale fluctuations, a high degree of localization is achieved to fluctuations very near the reflecting layer. This localization was used effectively in the detection of long wavelength Alfvén waves excited by fusion alpha particles in TFTR. [50]

\section{B. Correlation analysis}

We specify the density distribution by assuming a Gaussian correlation function of the form

$$
\frac{\langle n(z) n(z+\Delta z)\rangle}{n^{2}}=\left(\frac{\tilde{n}}{n}\right)^{2} C_{n} .
$$

where $C_{n}=\exp \left(-\Delta z^{2} / W_{n}^{2}\right) \cos \left(k_{z} \Delta z\right)$. Here $C_{n}$ is the normalized density correlation. Each solution of the wave solver incorporates a randomly distributed density fluctuation which conforms to this correlation function. Multiple random distributions are generated by the following prescription: Assume a white noise source $f(z)$. Then the spectrum of density fluctuations with the above correlation function is given by $N\left(k_{z}\right)=A^{1 /}\left(k_{z}\right) F\left(k_{z}\right)$ where $F\left(k_{z}\right)$ is the Fourier transform of $\mathrm{f}(\mathrm{z})$ and $A\left(k_{z}\right)$ is the Fourier transform of the correlation function in Eq. 15. The inverse Fourier transform of $N\left(k_{z}\right)$ gives only one realization of a random density distribution $\tilde{n}$ with the correlation property of Eq. 15 . A new realization $\tilde{n}(z)$ is generated for each solution of the 1-D wave equation. Several hundred such runs are performed for each probe frequency (i.e. position of the cutoff layer) in order to build up statistics for the correlation analysis. From the ensemble of runs for each frequency we can define a mean value for the reflected signal $\bar{U}_{\omega}=\left\langle U_{\omega}\right\rangle$ 
and the set of the perturbed field $\tilde{U}_{\omega}=U_{\omega}-\left\langle U_{\omega}\right\rangle$. Hence, $\left|\bar{U}_{\omega}\right|^{2}$ is the relative intensity of the coherent to total reflected signal.

The normalized cross correlation of the full wave solution is then given by

$$
C_{F . W .}\left(\omega_{1}, \omega_{2}\right)=\frac{\left\langle\tilde{U}_{\omega 1} \tilde{U}_{\omega 2}^{*}\right\rfloor}{\sqrt{\left\lfloor\tilde{U}_{\omega 1} \tilde{U}_{\omega 1}^{*} \tilde{\Lambda}^{\prime} \tilde{U}_{\omega 2} \tilde{U}_{\omega 2}^{*}\right\rfloor}}
$$

where the cutoff location $z_{c}(\omega)$ is a function of wave frequency. We also compute the normalized cross correlation using the geometric optics approximation to the outgoing solution

$C_{\text {G.o. }}\left(\omega_{1}, \omega_{2}\right)=\frac{\left\langle\tilde{G}_{\omega 1} \tilde{G}_{\omega 2}^{*}\right\rangle}{\sqrt{\left\lfloor\tilde{G}_{\omega 1} \tilde{G}_{\omega 1}^{*} \bigvee \tilde{G}_{\omega 2} \tilde{G}_{\omega 2}^{*}\right\rangle}}$

where $G=\exp (i \delta \phi), \delta \phi=\phi-\langle\phi\rangle$ and $\phi$ is given by Eq. 5. These correlation functions are then evaluated for a range of wave frequencies and over an ensemble of random density distributions.

\section{Validity of 1-D geometric optics for correlation analysis}

A key issue in reflectometry is the validity of the geometric optics in describing the correlation properties of the scattered field. We consider here how well geometric optics does in describing correlations in the radial direction in 1-D. Figure 9 shows the result of a correlation analysis for two different density correlation lengths $W_{n}=0.5 W_{\text {Airy }}$ and $W_{n}=10 W_{\text {Airy }}$. The fluctuation level is chosen so that the phase perturbation of the reflected signal is small $(\delta \phi<1)$.

In general the correlation lengths obtained from geometric optics are very similar to that obtained from 1-D full wave analysis. Surprisingly, even when $W_{n}<W_{\text {Airy }}$ as is the case 
shown in Figure 9a, the normalized correlations $C_{F . W .}, C_{G . o .}$ are almost identical, but differ substantially from $C_{n}$. For cases where $W_{n} \gg W_{\text {Airy }}$ the correlations $C_{\text {F.W. }}, C_{\text {G.o. }}$ are almost identical to each other and the similar to $C_{n}$.

In Figure 10 we plot the half widths $W_{0.5}^{F . W}, W_{0.5}^{G . O .}$ of $C_{F . W .}, C_{G . O}$. respectively against the half width $W_{0.5}^{n}$ of $C_{n}$ varying $W_{0.5}^{n}$ from 0.1 to $10 W_{\text {Airy }}$. The important result from this figure is that geometric optics is essentially identical to the full wave analysis for $W_{0.5}^{n} \geq 0.5 W_{\text {Airy }}$. For smaller values of $W_{0.5}^{n}$ neither $W_{0.5}^{F . W}, W_{0.5}^{G . O .}$ is a reliable measure of $W_{0.5}^{n}$. The resolution limit suggested by the 1-D is therefore approximately $W_{\text {Airy }}$.

\section{Numerical imaging of fluctuations in TFTR}

In the previous section it was shown that geometric optics provides an excellent approximation to the correlation analysis of reflectometry in 1-D provided the correlation length of the density fluctuations exceeds $W_{\text {Airy }}$. Recent laboratory experiments and simulation analysis at UCLA have shown that the 1-D full wave analysis of correlation reflectometry provides a good approximation to the observed correlation lengths in the Large Plasma Device (LAPD) although the range of observed correlation lengths is limited.[23] A natural question to ask is how well the random phase screen model (based on 1-D geometric optics) provides a description of scattering in 2-D. We now address the issue of whether the phase screen approximation provides a valid description of scattering near a cutoff layer in a fusion device by looking at properties of sideband correlations measured on the TFTR tokamak.

As a first step we take two time segments of X-mode reflectometer data on TFTR for discharge 65646 at two times $t=2.53$ and $t=2.7 \mathrm{sec}$ corresponding to the Ohmic and neutral beam heated phase of the discharge with cutoff profiles given in Fig. 4 for a wave frequency of $115 \mathrm{GHz}$. The distribution of the data in two $30 \mathrm{~ms}$ time segments is shown in figure 11 with the dashed curves representing lines of best fit using the Rice distribution. From the phase screen model, the best fit to the data in the Ohmic phase 
corresponds to an rms phase perturbation at the cutoff of $\sigma_{1}=0.5$ radians while in the neutral beam phase we can only say that $\sigma_{2}>2$ leading to a Rayleigh distribution for the amplitude fluctuations. For $\sigma<1$ where $\left\{E \boldsymbol{\rangle}=\exp \left(-\sigma^{2} / 2\right.\right.$, we can employ the method of sideband correlations to determine the validity of the phase screen approximation.

Figure 12a shows the spectrum $\left\{|E(\omega)|^{2}\right\rangle$ of reflectometer signals $E(t)$ detected during the Ohmic phase of the discharge. The high normalized sideband correlation $\left|C_{\omega,-\omega}\right|$ is shown in Figure $12 \mathrm{~b}$ where

$$
C_{\omega,-\omega}=\frac{\{E(\omega) E(-\omega)\rangle}{\left\langle|E(\omega)|^{2}\right\rangle} .
$$

A high degree of sidband correlation is indicative of scattering from a thin phase screen. The phase of the sidband correlation $\phi_{\omega,-\omega}$ is also shown in Figure 12c. The phase fits well to a parabola which suggests that $\omega \propto K_{\theta}$ and the phase shift is due to propagation away from the phase screen. A procedure for numerical back projection can be performed on the data by varying the degree of phase curvature in the sidband correlation. The phase of the sideband correlation will increase as the distance increases from the phase screen. A numerical projection is equivalent to the linear transformation $E(t) \rightarrow E^{\prime}(t)$ where

$$
E^{\prime}(t)=\int d \omega \exp (i \omega t) \cdot\left\{E(\omega) \exp \left(i a \omega^{2}\right)\right\}
$$

This is just the inverse transform of the Fourier transform of the detected signal $E(t)$ but with the addition of an extra phase curvature term $\exp \left(i a \omega^{2}\right)$ to effectively transform the plane of observation. It should be noted that this is precisely the action of a lens but where we are substituting $\omega$ for $\mathrm{k}$ by assuming that the fluctuations essentially drift rigidly across the detector.

Before proceeding to the numerical back projection of the TFTR data, we show a simulation of the amplitude spread of waves scattered from a thin phase screen. Figure 13 
shows a simulation of the distribution of data for scattering from a random phase screen. The distribution (histogram displayed as a contour plot) is shown for a range of distances from the phase screen. The spreading in amplitude and phase of the signal is shown with increasing distance from the screen from left to right. The essential point here is that as the waves propagate away from the screen then amplitude fluctuations develop and the distribution begins to rotate until it becomes circular and loses any indication of its original well ordered state. The transition from a well ordered phase modulated signal to a random amplitude and phase distribution on the right side of the figure is entirely the result of propagation. In the numerical back projection of the data, we look for signs of reduction in amplitude fluctuations and rotation of the distribution.

Figure 14 shows the result of a sequence of numerical back projections. Increasing the phase curvature of the sideband correlation in figure 12 by a large amount $z>2 \pi k_{0} / K_{\perp}^{2}$, leads to the circular distribution (frame 6). As the phase curvature is decreased, the circular distribution first becomes elliptic (frame 5) and then rotates, continuing to narrow in its amplitude spread until reaching the point of minimum amplitude fluctuations (frame 3). Beyond this range, the distribution again rotates and amplitude fluctuations increase, finally approaching more circular distribution. Figure 15 shows the amplitude distribution of the signals in frame 5 and frame 3 of Figure 14, indicating the amplitude compression occurring at the optimal (image) plane.

A reasonable question at this point is whether there is a significant difference in the properties of the phase of the signals between the two distributions with the largest and smallest amplitude spread. The answer is that there is a dramatic difference, as indicated in Figure 16. In particular, the phenomenon of phase jumps, which has plagued the quantitative interpretation of phase data for many years, is now conclusively shown to be strongly dependent of the propagation distance. Figure $16 \mathrm{c}$ shows the phase of the signal at maximum amplitude spread (corresponding to the distribution in frame 5 of Figure 14). The signal is full of phase jumps. The remedy up till now has been to surgically remove the jumps on the assumption that the period between the jumps is representative of the fluctuations in the medium. However, figure $16 \mathrm{~d}$ shows a small segment of the same data 
set in a narrow time window where there are no phase jumps. Instead we see phase spikes which are large transient phase events. These spikes could be misinterpreted as some intermittent turbulent activity instead of what it really is: an interference effect due to the spreading of the scattered waves from the cutoff layer. To make this clear, figure 16a and $16 \mathrm{~b}$ shows the phase of the same data for the case of numerical back projection (frame 3 of Figure 14) which corresponds to the case of minimum amplitude fluctuations. In this case we see that the large number of phase jumps in Figure 16c has mostly disappeared indicating that the jump feature is also a result of propagation away from the cutoff. Figure $16 \mathrm{~b}$ displays the phase for the same time window of the data shown in Figure 16d, indicating a signal where there is now an absence of phase spikes. Hence we can conclude that the spikiness of the phase data and the predominant number of phase jumps is essentially a result of interference effects resulting from the diffracted of waves propagating away from a mostly irregular phase screen. These results strongly suggest that imaging may indeed play a valuable role in reflectometry. The above analysis of TFTR data also suggests that the naïve approximation of geometric optics should not be underestimated in providing a description of the properties of reflectometer measurements in fusion plasmas. On the other hand, extensive multi-dimensional numerical simulation is required to understand better the limits of geometric optics and to deal with plasma and wavefront curvature, as well antenna and receiver geometry. In addition, extensive laboratory experiments are still needed to underpin theoretical studies.

\section{Recent developments and future directions}

In fusion science there is a pressing need to develop a quantitatively accurate description of reflectometry benchmarked against direct experimental measurements in a variety of scattering regimes. The objective is to transform the diagnostic from its historical role as an indicator of plasma fluctuations into a technique used to extract quantitatively accurate measurements of the statistical properties of plasma turbulence under a wide range of circumstances. In order to achieve this level of understanding, it is essential to develop a comprehensive description of the scattering process incorporating important experimental details such as mean plasma background variation, antenna geometry and the correlation 
properties of the plasma turbulence. In addition this modeling needs to incorporate and assess certain plasma processes such as resonant absorption and its possible role in the interpretation of the scattering data. A high level of simulation capability in turn requires well designed experiments which can measure the key plasma parameters including the fluctuations together with the scattered field in order to benchmark against numerical analysis. Such experimental studies are by no means straight forward given the difficulty of measuring all the relevant physical parameters of a plasma which impact the modeling of the scattered field such as the mean plasma profiles, fluctuation level and turbulence correlation length. In addition, the computational task of incorporating all these variables into a full wave solver is still a formidable task.

From a historical perspective the first attempts to address the interpretation of reflectometry rested on analysis of the 1-D full wave equation.[12-14,51] As pointed out above, the full wave equation reproduces the response of geometric optics for wavelengths greater than the Airy width but it does not explain the development of amplitude fluctuations in the reflected signal (except under conditions of wave absorption). The next step in the development of a description of reflectometry incorporated the 1-D geometric optics response locally with diffraction from a phase changing screen.[24,47] This model had the advantage of a quantitatively correct description of the plasma with the addition of phase dispersion for the scattered field which was shown to lead to amplitude fluctuations in early work done on the ionosphere.[6]

What was missing in these early studies was a description the real geometry of the experiment incorporating plasma background inhomogeneity with receiver and transmitter antenna structures. Within the context of scattering from a planar diffraction grating, the filtering effect of the antennas on the detected signal was studied extensively by a number of authors. [52-55] These studies revealed the intuitively reasonable result that the spot size of the transmitter/receiver determines its spectral response to fluctuation wavelength in the screen. The most recent development in this area, within the context of the phase screen model and full wave analysis, shows that the effect of plasma non- 
uniformity (specifically plasma curvature) leads to a much broader wavelength response for reflectometry than predicted from slab models.[56] Such developments clearly highlight the need for the inclusion of plasma inhomogeneities which can have a critical impact on the interpretation of experimental measurements.

The challenge of incorporating full wave analysis with plasma inhomogeneity and antenna structures has only recently been met with the advent of inexpensive parallel computers and algorithms allowing the Courant condition to be greatly exceeded, thus improving the efficiency of analysis.[57] Such developments allow for the first time the 2-D full wave simulation of correlation measurements using reflectometry, facilitating comparison to correlation properties of the measured signals. The result of these studies are now appearing more regularly in the literature, and hopefully these numerical studies will soon become common place in the investigation of reflectometry.

The studies discussed above all relate to the interpretation of reflectometer measurements in current experiments based on single receivers or a single receiver/transmitter system. However, it was argued here and elsewhere [24] that under certain conditions it may be possible to image fluctuations, and to recover the phase of geometric optics at the image plane using wide aperture optics. The possibility of imaging was further supported from statistical properties of data taken on TFTR [47] and by extensive numerical full wave analysis in plane wave slab geometry with 2-D fluctuations [58,59]. It is likely that the addition of detector arrays can be a valuable next step in reflectometry, particularly when coupled to a strong full wave simulation capability using the 2-D plasma equilibrium to help design experiments and interpret measurements. There are currently efforts under way to develop the technology needed to produce the first reflectometer images of fluctuations in fusion plasma experiments. [60] However, these developments are in themselves insufficient to place reflectometry on a firm foundation as a quantitative diagnostic of fluctuations in fusion plasmas. Only direct confirmation with experimental data can do this. There have been a number of successes in comparing measurements to model calculations in fusion scale experiments, and these efforts need to continue. $[22,23]$ 
Extensive data does exist on the comparison of reflectometer and probe measurements of plasma turbulence in the cold edge region of fusion plasmas and in basic plasma experiments where the insertion of probes deep into the plasma is possible, such as in the LAPD device at UCLA. Such studies have revealed a wealth of information on the measurement capability of reflectometry. Recent results from these investigations indicate that the magnetic field strength can be measured from the frequency difference between $\mathrm{O}$-mode and $\mathrm{X}$-mode reflectometry for the peak correlation due to turbulent fluctuations. Analysis based on 1-D full wave simulation is in good agreement with experiment on the LAPD device in a restricted range of parameters.

The sum total of all the work done in the basic science of reflectometer represents an impressive body of knowledge which has helped to provide a firm foundation for the quantitative interpretation of reflectometer measurements in the future. However, detailed numerical simulation is required to determine if such 1-D estimates can be extended to core fluctuation measurements in fusion plasmas. More basic studies are needed in order to continue to challenge present understanding and to refine theoretical models and simulation tools. As a case in point, the role of resonant absorption by wave tunneling beyond the cutoff layer has been proposed as playing a role in some experiments, however this line of research has not been actively pursued. In fusion plasmas, the most relevant resonance involves the upper hybrid frequency which is close to the cutoff layer at low density near the plasma edge for the upper X-mode. A direct measurement of the wave field in the presence of this resonance and comparison of fluctuation measurements to full wave simuations incorporating the effect of resonant absorption is still needed. A further area where analysis is required is the effect of large density fluctuation levels in the edge plasma on reflectometer measurements. Both geometric optics and 1-D full wave analysis indicate that weak scattering can still occur from large density fluctuation levels at the plasma edge, primarily due to the short density scale length and long wavelength of the microwave beam needed for low edge density studies. Such a systematic analysis can readily be performed on existing experiments. 
In the area of imaging of fluctuation, it is clear that measurement of the complex electric field is needed in order to extract the phase and amplitude fluctuations at the image plane. At present, not all experiments measure the complex amplitude, so more effort is required to extract all the available information from reflectometer fluctuation measurements. The first demonstration of the validity of the phase screen description for scattering near a cutoff actually occurred in space science using ionosonde measurements of the ionosphere.[49] These early studies showed that the rotation of the complex amplitude of the electric field as predicted by the model was actually consistent with experimental data from F-layer measurements. The first confirmation of the same result, with the addition of numerical back projection, was performed on TFTR.[48] It is appropriate that this line of investigation be pursued by implementing an array of detectors and heterodyne receivers to study the potential for imaging.

Reflectometry has already had a major impact on our understanding of the relation between turbulence and transport in fusion plasmas. The technique in combination with other diagnostics has shed valuable new light on the behavior of plasma instabilities, improving their characterization and addressing their correlation with plasma performance. Three major areas where reflectometery is yielding valuable results are in RF wave physics of heating and current drive, energetic particle driven instabilities and turbulence behavior in the transition to enhanced energy confinement regimes. [32-39,50]

The future development of the field will require very close coupling between theory, simulation capability and experiment. The role of simulation in 2-D and even 3-D cannot be underestimated in providing new insight into the method and its uses. Visualization of the scattering process will be a powerful tool in aiding the conceptualization of the physics of reflectometry and will greatly assist in the development of new and exciting diagnostic ideas. From the experimental perspective, a more detailed and focussed effort is required to map the field distribution at the plasma boundary for comparison with full wave calculations. This includes the detection of the complex amplitude and the use of multiple detectors to understand the angular spread and deflection of the scattered radiation. 


\section{Acknowledgments}

The authors have greatly benefited from numerous discussions with friends and colleagues in the reflectometer community. However the views and any errors expressed in the paper are entirely the responsibility of the authors. Special thanks go to Jim Irby and Yijun Lin at MIT, Tony Peebles, Edward Doyle, Mark Gillmore and Terry Rhodes at UCLA, Garrard Conway at Max Plank Garching. Thanks also to Ken Young for his encouragement and support. This work is funded by DoE contract No. DE-AC02$76 \mathrm{CH} 03073$. 


\section{References}

[1] K. Bibl, Annali di Geofisica, 41, 667 (2000)

[2] J.A. Ratcliffe, Nature, No. 4105, p.9 (1948)

[3] J.W. Wright, P.E. Argo and M.L.V. Pitteway, Radio Science 31, 349 (1996).

[4] S.A. Bowhill, J. Atm. Terr. Phys. 11 p.91 (1957)

[5] H.G. Booker, J.A. Ratcliffe and D.H. Shinn, Phil. Trans. Roy. Soc. A, 242 p.575 (1950)

[6] A. Hewish, Proc. Roy. Soc. A 209, 81 (1951).

[7] J.A. Fejer, Proc. Roy. Soc. A, 220 p.455 (1953)

[8] R.P. Mercier, Camb. Phil. Soc. 58 p.382 (1962)

[9] H.G. Booker, P.K. Pasricha and W.J. Powers, J. Atm. Terr. Phys. 48, 327 (1986).

[10] M.L.V. Pitteway, Proc. Roy. Soc. A 252, 556 (1958).

[11] M.L.V. Pitteway, Proc. Roy. Soc. A 253, 86 (1959).

[12] E. Mazzucato and R. Nazikian, Plas. Phys. Cont. Fusion 33, 261 (1991).

[13] N. Bretz, Phys. Fluids B 4, 2414 (1992).

[14] I.H. Hutchinson, Plasma Phys. Cont. Fusion 34, 1225 (1992).

[15] B.I. Cohen, E.B. Hooper, M.C. Spang and C.W. Domier, Rev. Sci. Instrum. 70, 1407 (1999).

[16] B.I. Cohen, L.L. LoDestro, E.B. Hooper and T.A. Casper, Plasma Phys. Cont. Fusion 40, 75 (1998).

[17] A.J.H. Donné, M. de Baar and R. Cavazzana, Rev. Sci. Inst. 68, 473 (1997).

[18] C.A. Hugenholtz, J.H. Donné, B.S.Q. Elzendoorn et al., Rev. Sci. Inst. 70, 1034 (1999).

[19] A.E. Costley, P. Cripwell, R. Prentice and A.C.C. Sips, Rev. Sci. Inst. 61, 2823 (1990).

[20] E.J. Doyle, T.L. Rhodes, W.A. Peebles and L. Zeng, Rev. Sci. Inst. 70, 1064 (1999). 
[21] M. Manso, F. Serra, B. Kurzan et al., Plasma Phys. Cont. Fusion 40, 747 (1998).

[22] M. Gilmore, W.A. Peebles and X.V. Nguyen, Plasma Phys. Control. Fusion 42, 655 (2000).

[23] M. Gilmore, W.A. Peebles and X.V. Nguyen, Rev. Sci. Inst. 72, 293 (2000).

[24] R. Nazikian and E. Mazzucato, Rev. Sci. Inst. 66 p.392 (1995).

[25] T.L. Rhodes, W.A. Peebles, E.J. Doyle et al., Plasma Phys. Control. Fusion 40, 493 (1998).

[26] M. Gilmore, W.A. Peebles and X.V. Nguyen, Plasma Phys. Control. Fusion 42, L1 (2000).

[27] T.L. Rhodes, S. Baang, A.E. Chou et al., Rev. Sci. Inst. 63, 4599 (1992).

[28] P. Cripwell, A.E. Costley and A.E. Hubbard, Proceedings, $16^{\text {th }}$ European Physical Society Conference on Plasma Physics and Controlled Fusion, Venice, 1989, (EPS, 1989) Vol. 1, p. 75.

[29] A.E. Costley, P. Cripwell and G. Vayakis, in Local Transport Studies in Fusion Plasmas, edited by J.D. Callen, G. Gorini and E. Sindoni, (SIF, Bologna, 1993).

[30] V.A. Vereshkov, V.V. Dreval and S.V. Soldatov, Rev. Sci. Inst. 70, 1700 (1998).

[31] K. Shinohara, R. Nazikian, T. Fujita et al., Rev. Sci. Inst. 70, 4246 (1999).

[32] E. Mazzucato, S.H. Batha, M. Beer et al., Phys. Rev. Lett 77, 3145 (1996).

[33] E.J. Synakowski, S.H. Batha, M.A. Beer et al., Phys. Rev. Lett. 78, 2972 (1997).

[34] G.D. Conway, D.N. Borba, B. Alper et al., Phys. Rev. Lett. 84, 1463 (2000).

[35] R. Nazikian, K. Shinohara, R. Yoshino et al., Proceedings of the $17^{\text {th }}$ International Atomic Energy Agency Fusion Energy Conference, Yokahama, 1998, (IAEA, Vienna, 1999) Vol. 4, p. 1689.

[36] K. Shinohara, R. Yoshino, R. Nazikian et al., Proceedings, $26^{\text {th }}$ European Physical Society Conference on Plasma Physics and Controlled Fusion, Maastricht, 1999, (EPS, 1999). 
[37] E. Doyle, et al., Proceedings of the $18^{\text {th }}$ International Atomic Energy Agency Fusion Energy Conference, Sorrento, 2000, (IAEA, Vienna, 2001) to be published in Nuclear Fusion.

[38] C.L. Rettig, W.A. Peebles, E.J. Doyle et al., Phys. Plasmas 4, 4009 (1997).

[39] C.M. Greenfield, J.C. DeBoo, T.C. Luce et al., Phys. Plasmas 7, 1959 (2000).

[40] R.E. Barrington, in Physics of the Earth's Upper Atmosphere, edited by C.O. Hines, I. Paghis, T.R. Hartz and J.A. Fejer, (Prentice-Hall Inc., Edgewood Cliffs. N.J., 1965)

[41] H. Bindslev, Plasma Phys. Cont. Fusion 33, 1775 (1991).

[42] J. Egedal and H. Bindslev, Plasma Phys. Cont. Fusion 35, 543 (1993)

[43] E. Mazzucato, Phys. Plasmas B4, 3460 (1992)

[44] H. Bindslev, Plasma Phys. Cont. Fusion 35, 1093 (1993).

[45] R. Hawryluk, Plasma Phys. Controlled Fusion 33, 1509 (1991).

[46] E.J. Doyle, T.L. Rhodes, J.L. Doane and W.A. Peebles, Rev. Sci. Inst. 66, 1233 (1995).

[47] E. Mazzucato and R. Nazikian, Phys. Rev. Lett. 71, p.1840 (1993)

[48] R. Nazikian, J. Mod. Opt. 44, 1037 (1997).

[49] R.P. Mercier, Cam. Phil. Mag. A ( $8^{\text {th }}$ Series) 4, 763 (1959). R.P. Mercier, Cam. Phil. Soc. 58, 382 (1962).

[50] R. Nazikian, G.Y. Fu, S.H. Batha, et al., Phys. Rev. Lett. 78, 2976 (1997).

[51] B.B. Afeyan, A.E. Chou, B.I. Cohen, Plasma Phys. Cont. Fusion 37, p.315 (1995)

[52] G.D. Conway, Plasma Phys. Cont. Fusion 39, 1261 (1997).

[53] G.D. Conway, Plasma Phys. Cont. Fusion 39, 407 (1997).

[54] E. Holtzhauer, M. Hirsch, T. Grossmann et al., Plasma Phys. Cont. Fusion 40, 1869 (1998).

[55] V. Zhuravlev, J. Sanchez and E. de la Luna, Plasma Phys. Cont. Fusion 38, 2231 (1996). 
[56] Y. Lin, R. Nazikian, J.H. Irby, E. Marmar, Plasma Physics and Controlled Fusion 43, L1 (2001).

[57] H.X. Vu, J. Comp. Phys. 144, 257 (1998).

[58] E. Mazzucato, Rev. Sci. Inst. 69, 1691 (1998).

[59] E. Mazzucato, Rev. Sci. Inst. 69, 2201 (1998), see also PPPL Report 3408.

[60] R.P. Hsia, B.H. Deng, W.R. Geck et al., Rev. Sci. Inst. 68, 488 (1997). 


\section{Figure Captions}

Figure 1

The coordinate system used in Eq. 1 and Eq. 2, with the wave vector pointing in the zdirection and the magnetic field in the z-y plane.

Figure 2

The square of the refractive index for four angles of incidence $\left(90^{\circ}, 80^{\circ}, 70^{\circ}, 60^{\circ}\right)$ to the magnetic field is plotted against $X=\left(\omega_{p e} / \omega\right)^{2}$ for (a) $Y=\omega_{c e} / \omega=0.7$ for propagation above the electron cyclotron frequency and (b) $Y=\omega_{c e} / \omega=1 . \therefore$ corresponding to propagation below the electron cyclotron frequency. The $\mathrm{O}, \mathrm{RX}$ and LX refractive index curves are marked. The polarization of the O-mode is basically parallel to the B-field whereas the RX and LX modes are perpendicular to the magnetic field. Both the RX and the O-X cutoffs $(\mu=0$ crossing) are accessible from the plasma edge where $\mu=1$, however the LX cutoff is not accessible for $Y<1$. In contrast, (b) shows that the LX and $\mathrm{O}$ mode cutoffs are accessible from the plasma edge when $\omega<\omega_{c e}$.

Figure 3

The polarization or degree of ellipticity $|\mathrm{R}|$ for the O-mode is indicated for the four angles of incidence to the magnetic field $\left(90^{\circ}, 80^{\circ}, 70^{\circ}, 60^{\circ}\right)$. The figure indicates increasing ellipticity with decreasing angle of incidence for the cases in Figure 2. The polarization for the $\mathrm{X}$-mode is complementary to the O-mode polarization and is not shown.

Figure 4

Profiles (a) of the RX, LX and O-mode cutoff layers vs major radius along the plasma midplane for an Ohmic TFTR plasma (\#65610 $\mathrm{t}=2.53 \mathrm{sec})$ with the following parameter: Toroidal magnetic field $B_{T}=4.5 \mathrm{~T}$, central electron density $\mathrm{n}_{\mathrm{e}}(0)=2 \times 10^{13} \mathrm{~cm}^{-3}$, plasma current $\mathrm{I}_{\mathrm{p}}=1.1 \mathrm{MA}$, plasma major radius $\mathrm{R}=2.6 \mathrm{~m}$. Profiles of the electron density and magnetic field strength on the plasma midplane are shown in (b). These curves represent propagation 90 degrees to the magnetic field. The location of the upper hybrid resonance is the curve lying between the electron cyclotron and the RX cutoff. 
Figure 5

The trajectory of $\mathrm{X}$-mode rays for four angles of inclination to the $\mathrm{X}$-axis $\left(89^{\circ}, 80^{\circ}, 70^{\circ}\right.$, $60^{\circ}$ ) and for two orthogonal orientations of the magnetic field. The density varies linearly in the vertical direction $\left(90^{\circ}\right.$ inclination) and $\mathrm{Y}=0.7$ for reflection from the $\mathrm{RX}$ cutoff. The magnetic field is coming out of the page in the upper set of curves and is in the direction of the tilted rays in the lower set of four trajectories.

Figure 6

In (a) a schematic of a reflectometer experiment showing the transmitter and receiver and the reflecting layer perturbed by turbulent eddies (gray blobs). In (b) a schematic of a generic detection scheme showing transmitter, receiver, local oscillator and basic analysis of the complex signals. The signals are in general complex, $E(t)=E_{\operatorname{Re} a l}+i E_{\operatorname{Im} a g}=A(t) e^{i \phi(t)}$. Simultaneous reflections from different cutoff layers are recorded and the signals are numerically correlated to determine the radial scale length of the blobs.

Figure 7

Plot (a) indicates waves scattered from a sinusoidal phase screen showing the formation of two symmetric sidebands. The difference in the parallel wave number between the two forward scattered sidebands from a thin sinusoidal perturbation of wavenumber $K_{x}$ and the incident wave is given by $K_{x}^{2} / 2 k_{0}$. This difference in wavenumber leads to a relative rotation of the complex amplitude of the scattered field relative to the unscattered field as the waves propagate away from the phase screen shown in (b) where the rotation angle is given by $\Delta \theta=z K_{x}^{2} / 2 k_{0}$.

\section{Figure 8}

The full wave solution of the wave field for $115 \mathrm{GHz} \mathrm{X}$-mode launch at the outer midplane for the discharge of figure 4 along with the electron density profile in (a). The location of the reflection point is indicated by the position of the last fringe of the standing wave pattern at around $3.1 \mathrm{~m}$ where the waves propagate in from right to left. 
The width of the last fringe is typically given by the Airy width, $W_{\text {Airy }}=0.5 L_{\varepsilon}^{1 / k_{0}^{2 / 3}}$ where $L_{\varepsilon}$ is the scale length of the permittivity at the cutoff. An initial density perturbation $\tilde{n}_{e}=a \cdot \exp \left[\left(z-z_{0}\right)^{2} / W^{2}\right] \cos \left[K_{z}\left(z-z_{0}\right)\right]$ is used to test the reflectometer response. In (b) the phase of the full wave solution of the outgoing field is plotted (dashed) as a function of the center of the density perturbation $z_{0}$ for parameters $a=10^{-3} n_{e}, W=0.5 W_{\text {Airy }}$ and $K_{z}=8 \mathrm{~cm}^{-1}$. In (c) the parameters for the density perturbation are $a=10^{-3} n_{e}, W=10 W_{\text {Airy }}$ $K_{z}=2 \mathrm{~cm}^{-1}$.

\section{Figure 9}

Simulation of correlation reflectometry for a model linear density profile with density scale length $L_{n}=5 \mathrm{~cm}$, density fluctuation level $\tilde{n} / n=10^{-3}$, and incident wave vector $k_{0}=25 \mathrm{~cm}^{-1}$. These parameters were chosen to correspond to conditions similar to edge plasma measurements in a fusion plasma. In (a) a short density correlation length is used, where the 1/e width $\left(W_{n}\right)$ of the density correlation is half the Airy fringe width $\left(W_{n}=0.5 W_{\text {Airy }}\right)$. Plotted is the normalized density correlation $C_{n}$ as a function of the displacement of the cutoff layers. Also shown is the normalized correlation of the outgoing full wave solution $C_{F . W}$. and outgoing geometric optics approximation to the field $C_{G . O}$ as a function of the separation of the cutoff layers. Each point in the correlation represents an ensemble average of the solution of the wave equation for 200 random density distributions. In (b) a density correlation length $W_{n}=10 W_{\text {Airy }}$ is used.

\section{Figure 10}

Comparison of the half width of the density correlation $W_{0.5}^{n}$ used in the full wave analysis in Figure 9 and the half width of the normalized correlation of the full wave solution $W_{0.5}^{F . W .}$. Also shown is the correlation based on the approximation of geometric optics $W_{0.5}^{G . O .}$. These parameters are plotted in ratio to the Airy fringe width $W_{\text {Airy }}$. The half width of the density correlation is scanned from 0.1 to $10 W_{\text {Airy }}$. Geometric optics and full wave analysis are in excellent agreement for correlation lengths down to 0.5 Airy fringes. 
Figure 11

Histogram distribution of two reflectometer signals $E=E_{R}+i E_{I}=A \exp (i \theta)$ taken on a $120 \mathrm{GHz}$ X-mode reflectometer channel during ohmic operation $\left(\sigma_{1}\right)$ and during neutral beam heating $\left(\sigma_{2}\right)$ in the discharge of Figure 4. Shown in (a) are curves of best fit using a Rice distribution for $E_{R}$, (the real part of the distribution where $\left\langle E_{I}\right\rangle=0$ ); in (b) is plotted the wave amplitude $\mathrm{A}$ and $\theta$ (the phase distribution) is shown in (c). The two distributions correspond to weak irregularities $\left(\sigma_{1}=0.5\right)$ and strong irregularities $\left(\sigma_{2}>2\right)$ for the ohmic and neutral beam phase of the discharge, respectively where $\sigma$ is the rms phase of geometric optics.

Figure 12

The spectrum $\left\langle|E(\omega)|^{2}\right\rangle$ in (a) of the complex signal $E(t)$ of the ohmic data in Figure 11. The high normalized sideband correlation $\left|C_{\omega,-\omega}\right|$ is shown in (b) and the phase of the sideband correlation $\phi_{\omega,-\omega}=\arg \left(C_{\omega,-\omega}\right)$ is shown in (c). The phase is fit well with a parabola, suggesting that $\omega \propto K_{\theta}$ and the sideband phase is due to propagation away from the cutoff layer, which varies with distance like $z K_{\theta}^{2} / 2 k_{0}$.

Figure 13

Simulation of the histogram distribution if the complex amplitude of the field scattered by a random phase screen. The histogram is presented as a contour plot where the largest number of points correspond to the inner most contours. The spreading in amplitude of the complex signal increases with increasing distance from the screen indicated by the progression of the distribution from left to right. As the waves propagate away from the screen the signal picks up amplitude spread and the distribution tilts relative to the origin, finally becoming circular (uncorrelated real and imaginary components).

Figure 14

The distribution of the signal used in Figure 12 as a result of a sequence of back projections for data taken between $\mathrm{t}=2.53$ to $\mathrm{t}=2.59 \mathrm{sec}$ in the ohmic phase of the discharge. The back projection is equivalent to modifying the parabolic curvature of the 
phase of the sideband correlation in Figure 12. The sequence of increasing back projection distance is indicated in the frames from 1 to 6 . The distribution of frame 6 corresponds to a projection far from the cutoff layer where the distribution becomes circular. As the signals are propagated numerically towards the cutoff layer the distribution becomes more elliptic and eventually rotates to assume a predominantly phase modulated form in frame 3. As the projection moves beyond the point of optimal imaging, the distribution again rotates and the amplitude spread increases.

\section{Figure 15}

A plot (a) of the amplitude distribution of the signal in frame 3 of Figure 14 compared to (b) the amplitude distribution of the same data in frame 5 of Figure 14. The difference in the amplitude spread of the signals indicates the degree of amplitude compression achieved by numerical imaging.

\section{Figure 16}

Shown in (a) and (c) is the phase of the signal at the numerical image plane and the phase far from the image plane at the point of maximum amplitude fluctuations, respectively. In (c) the data is full of phase jumps. A smaller segment of data is shown in (d) where there are no phase jumps. Instead large intermittent phase spikes are observed. In (a) the phase of the same data is shown at the numerical image plane. Phase jumps are minimized. In (b) for the same small time window as in (d) the strong phase spikes are also not apparent. The removal of the phase jumps and phase spikes has been achieved by numerical back propagation, without the need to selective phase jump removal methods. 


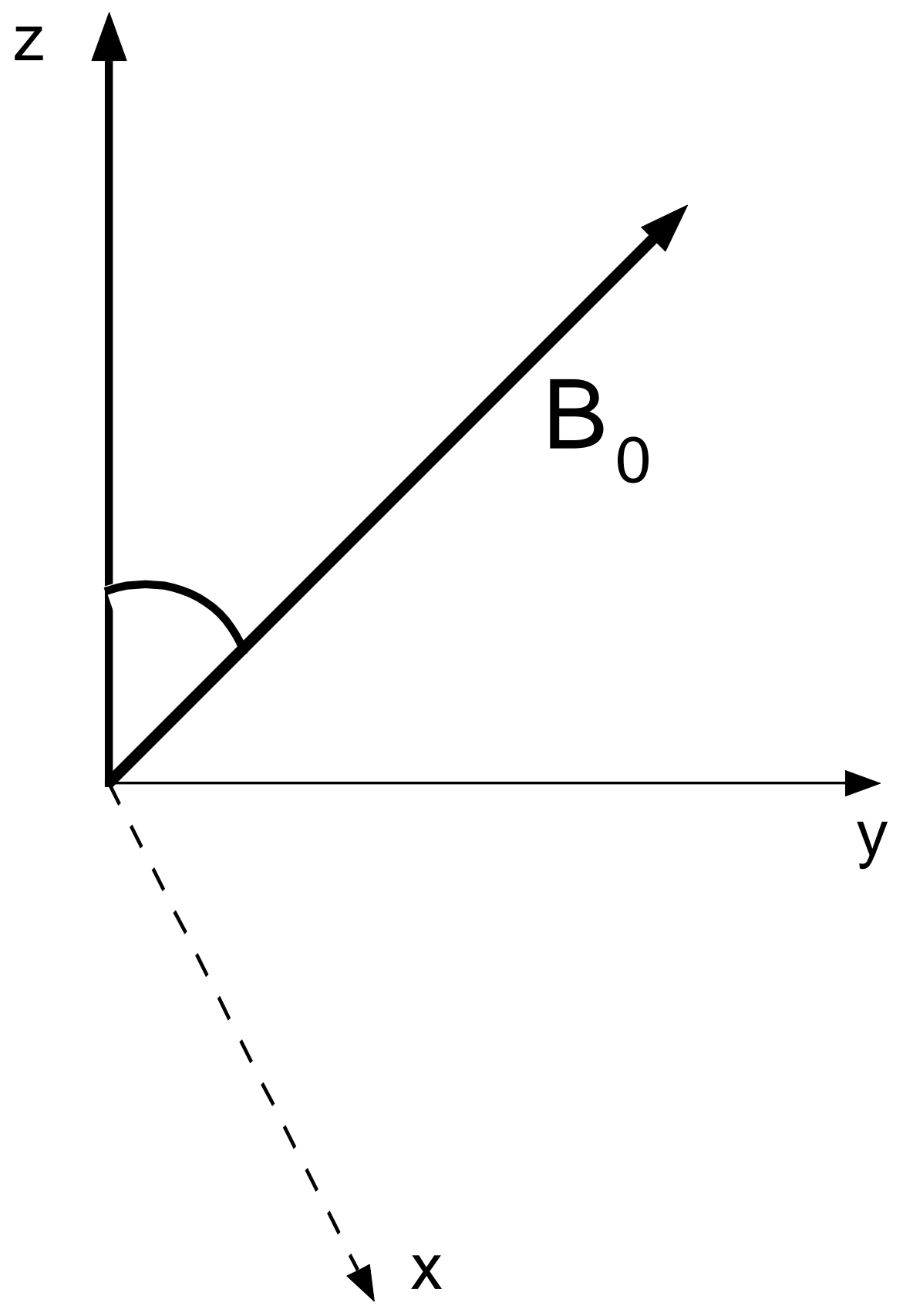

Figure 1

R. Nazikian 

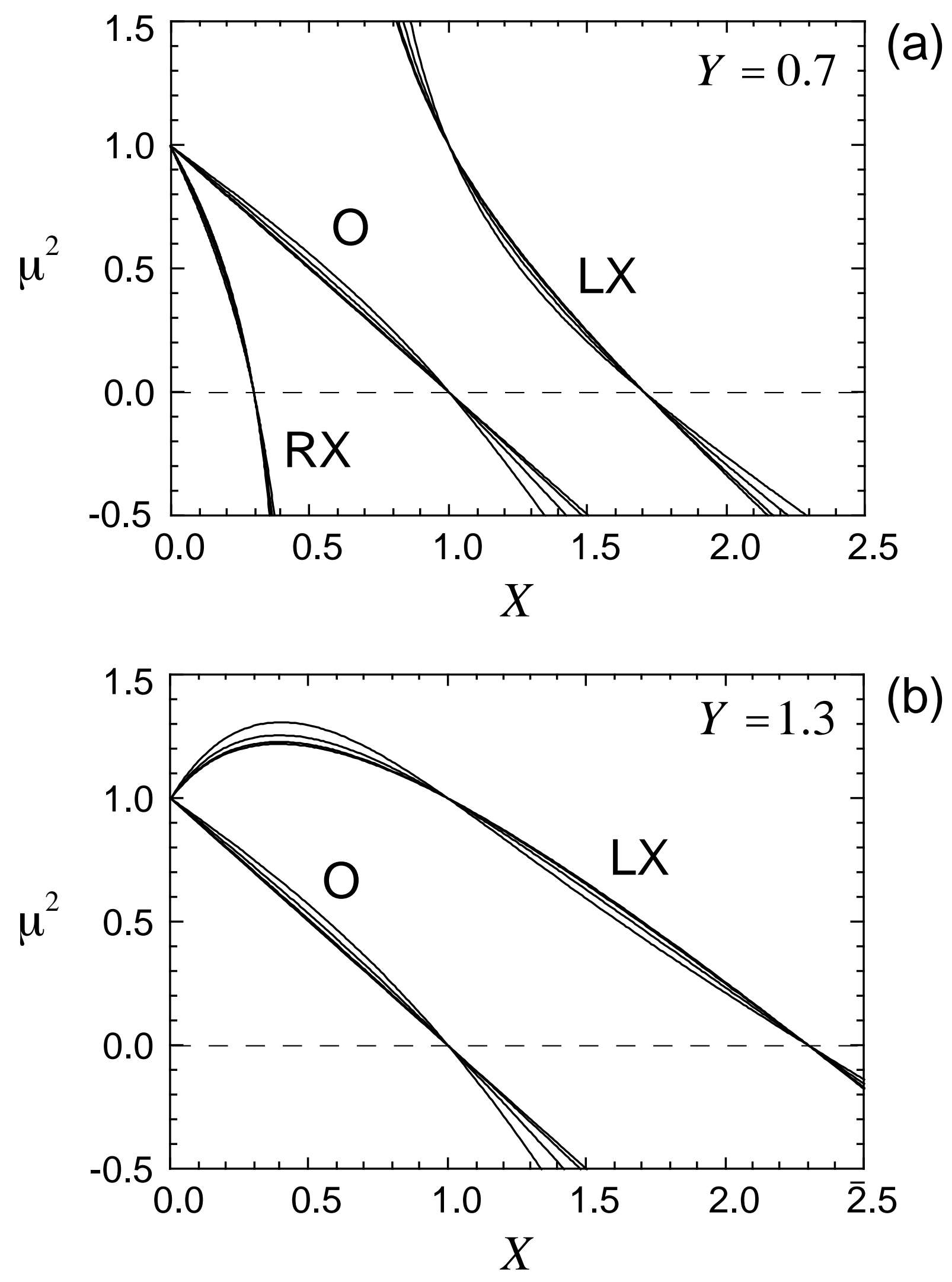

Figure 2

R. Nazikian 


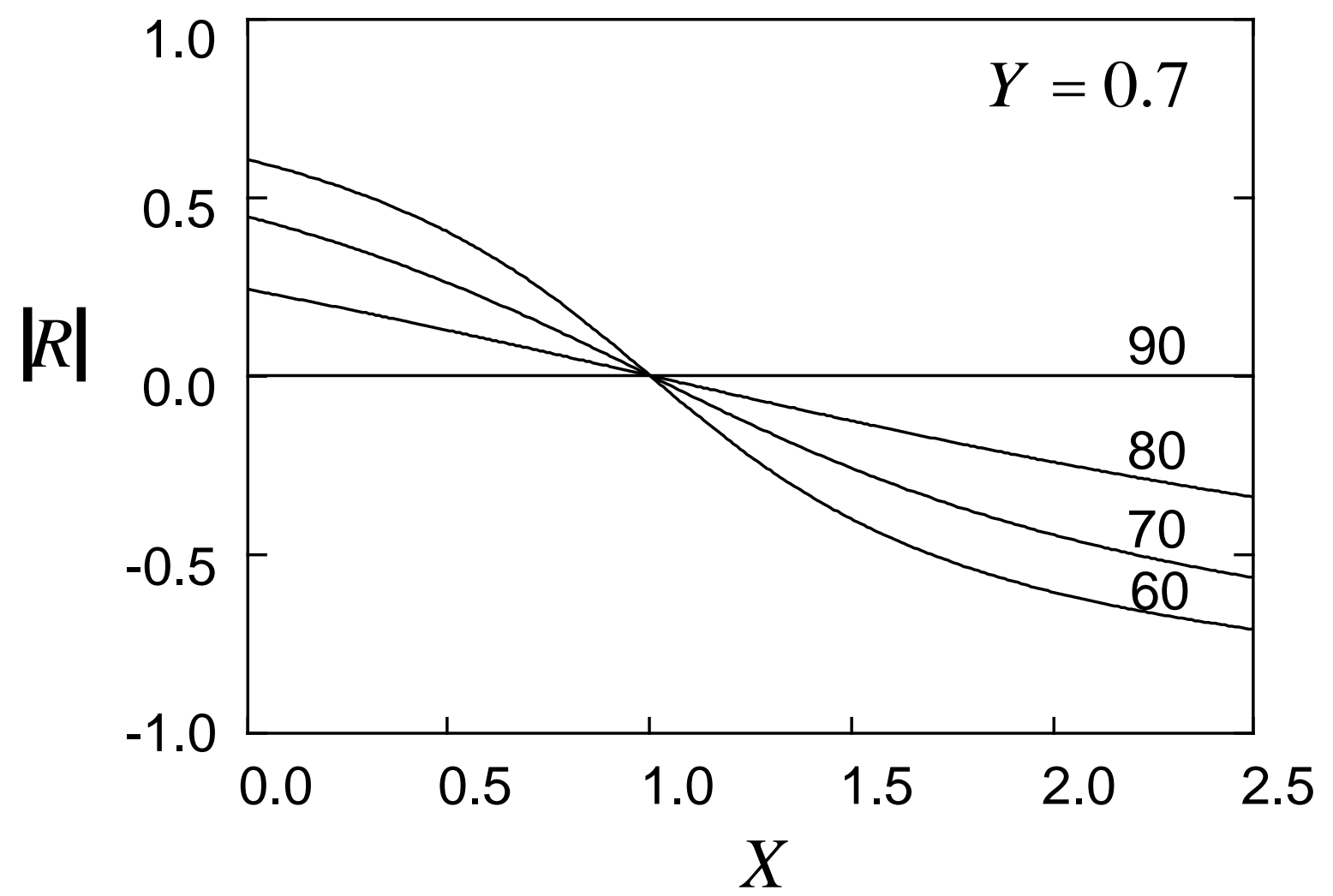

(a)

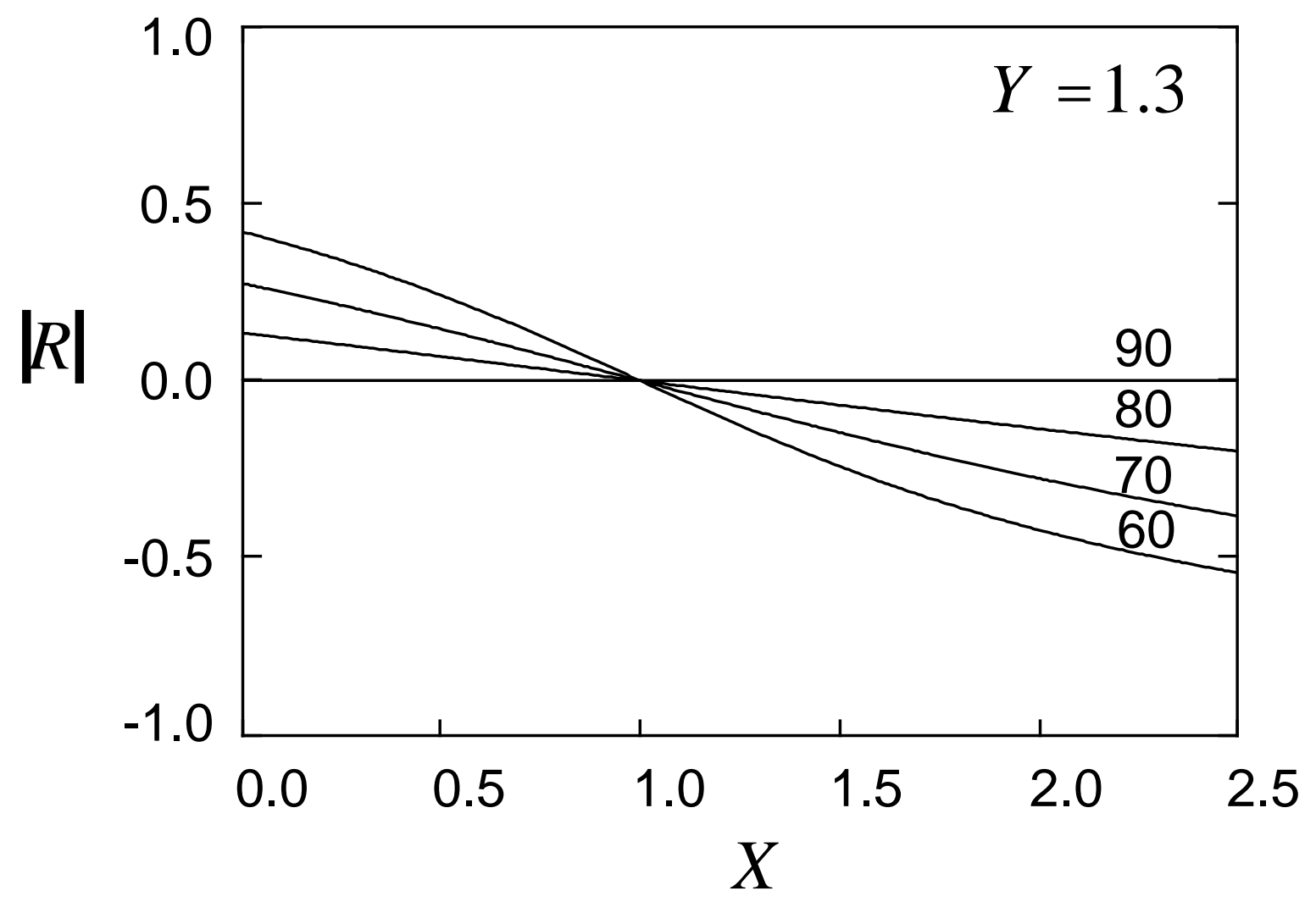

(b)

Figure 3

R. Nazikian 


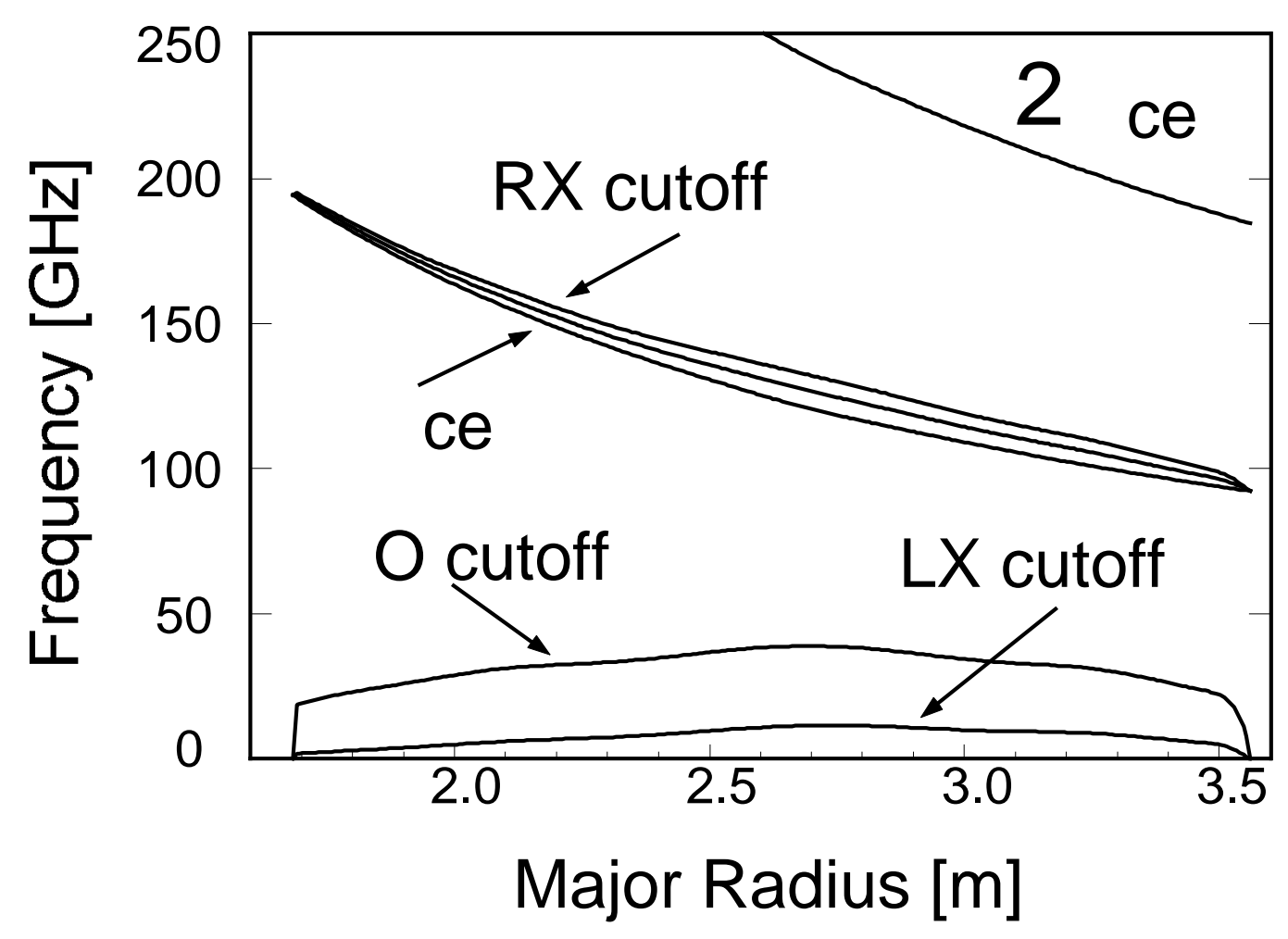

(a)

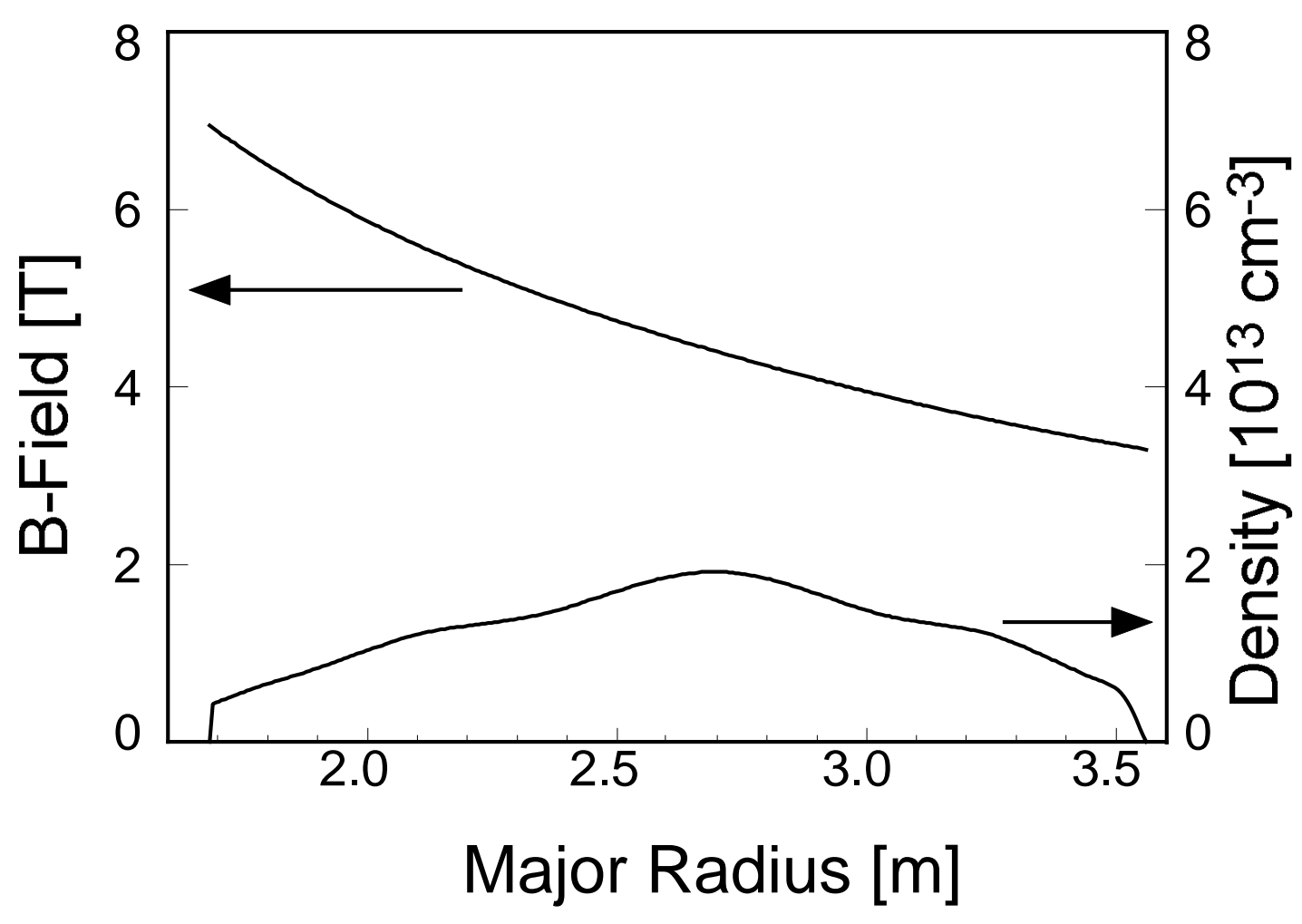

(b)

Figure 4

R. Nazikian 


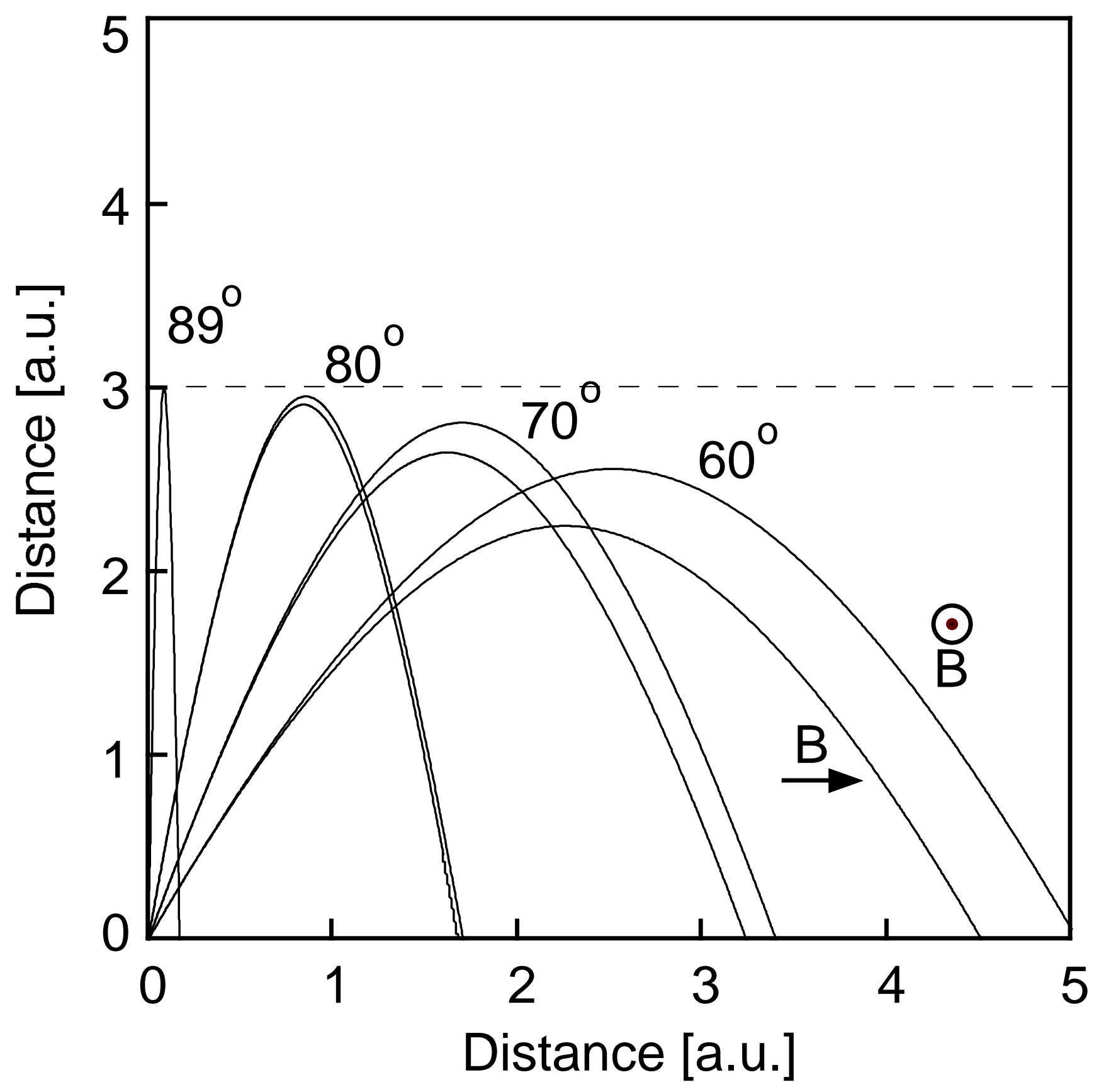

Figure 5

R. Nazikian 
(a)
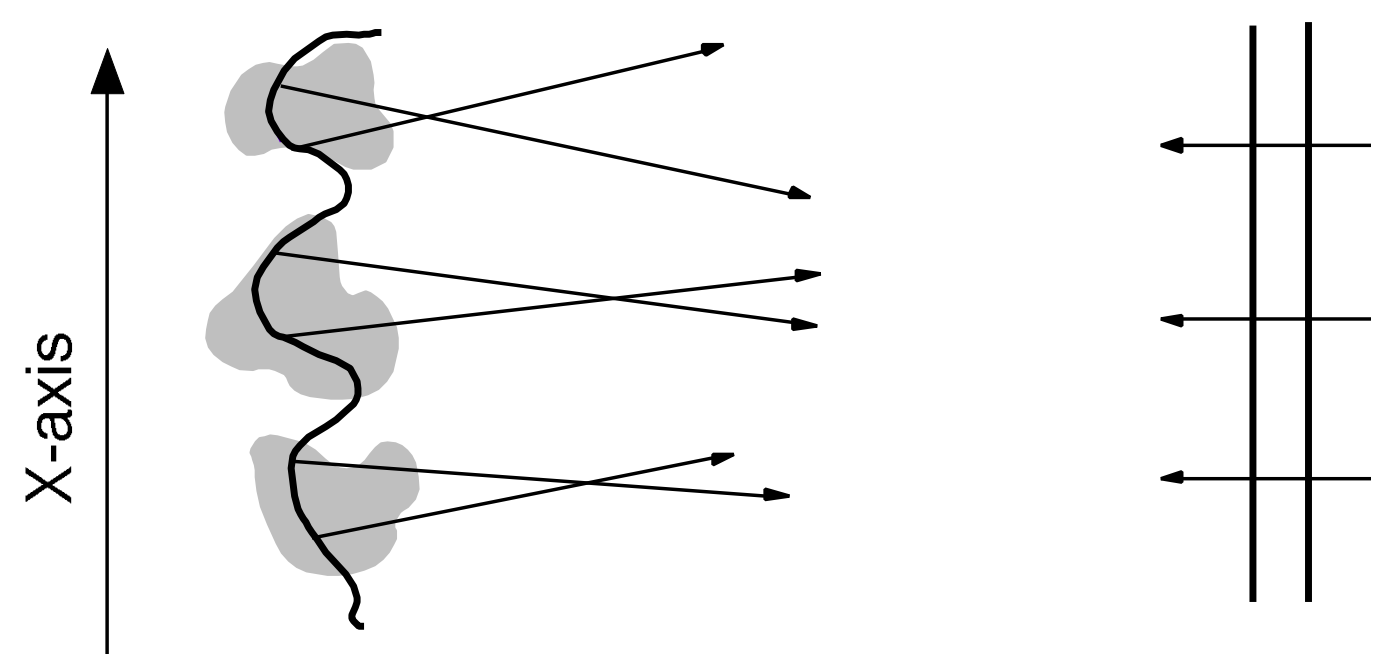

Transmitter
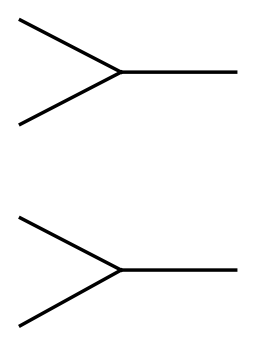

Receiver

\section{z-axis}

(b)
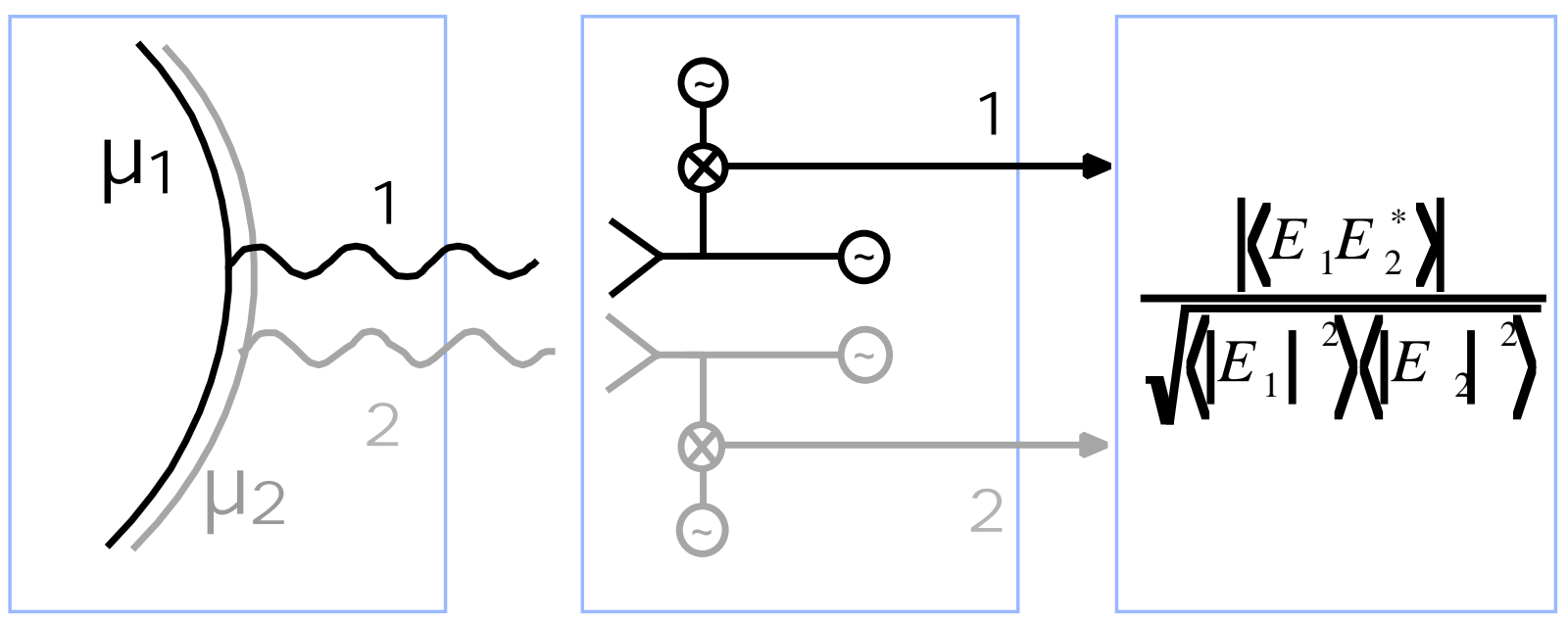

Figure 6

R. Nazikian 


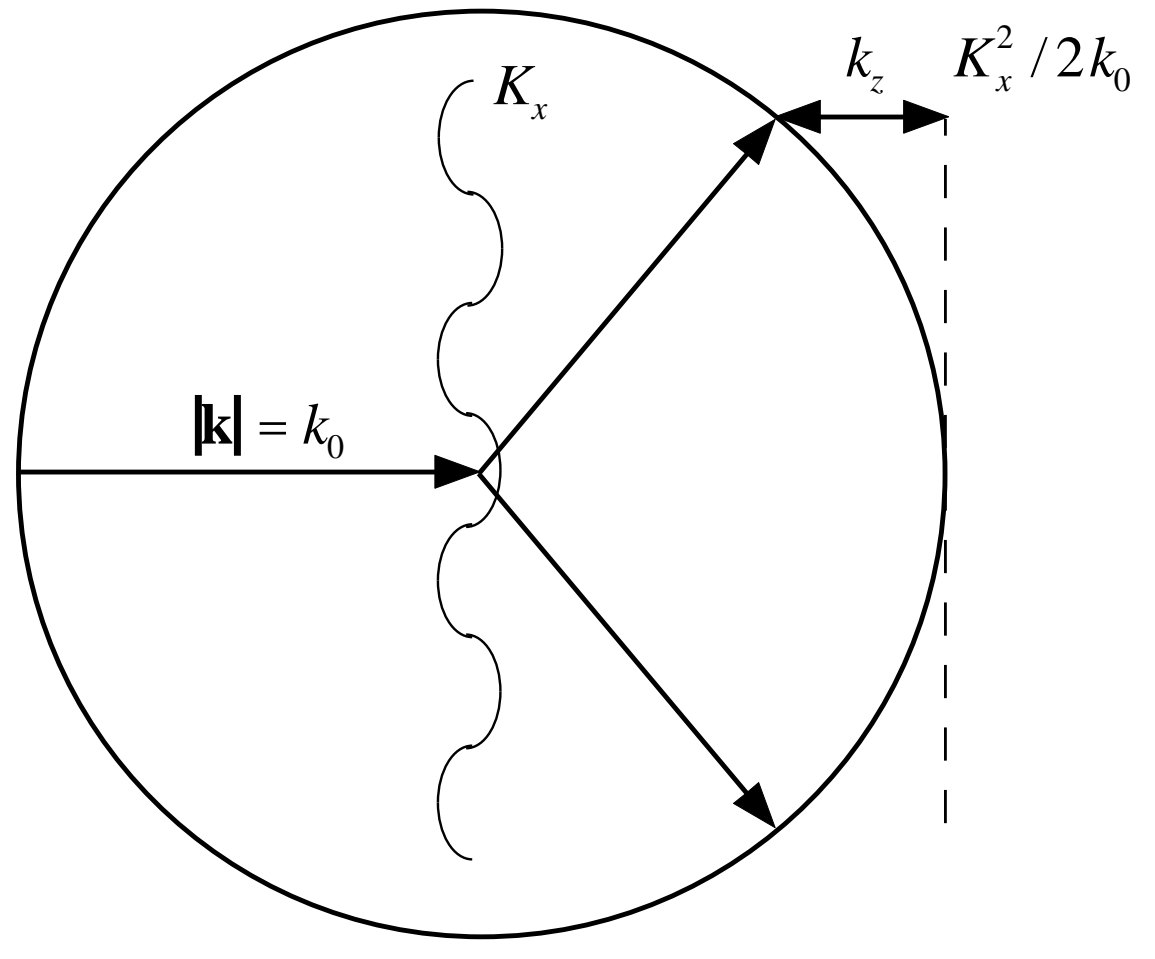

(a)

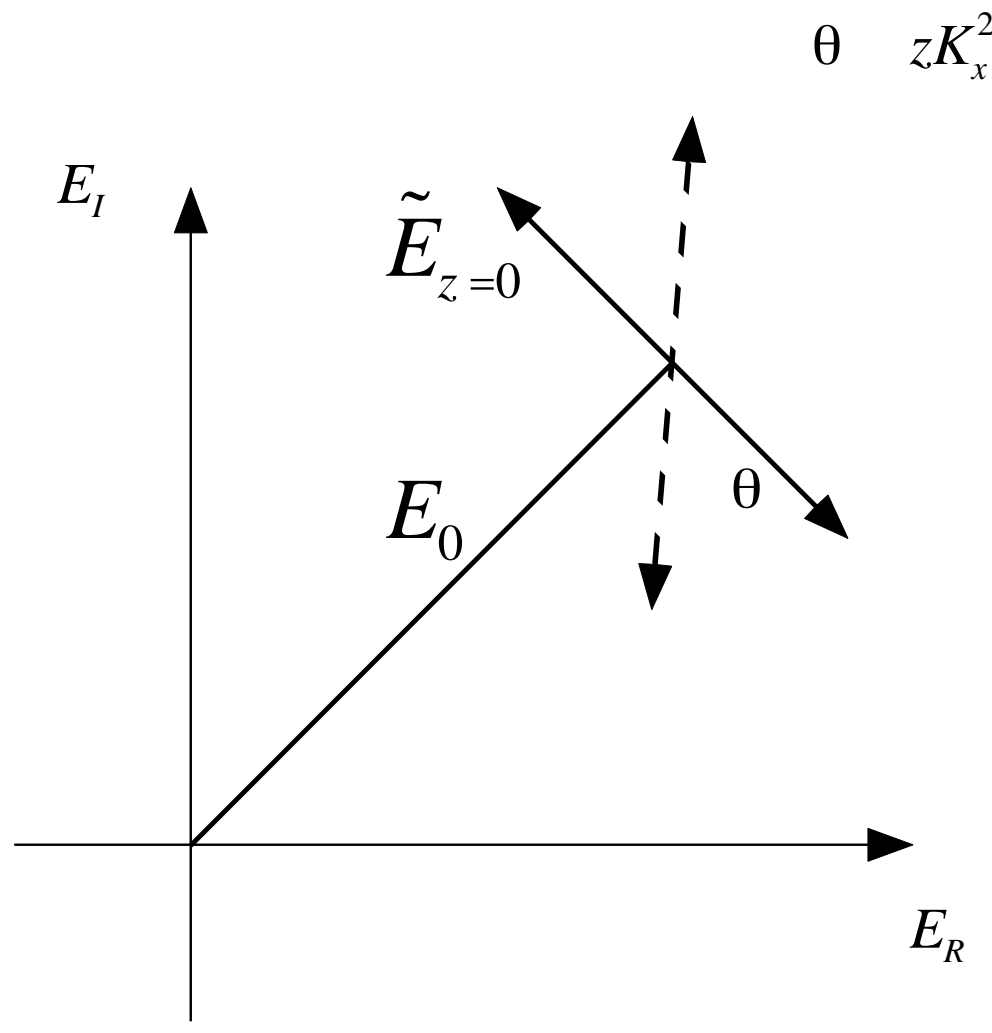

(b)

Figure 7

R. Nazikian 


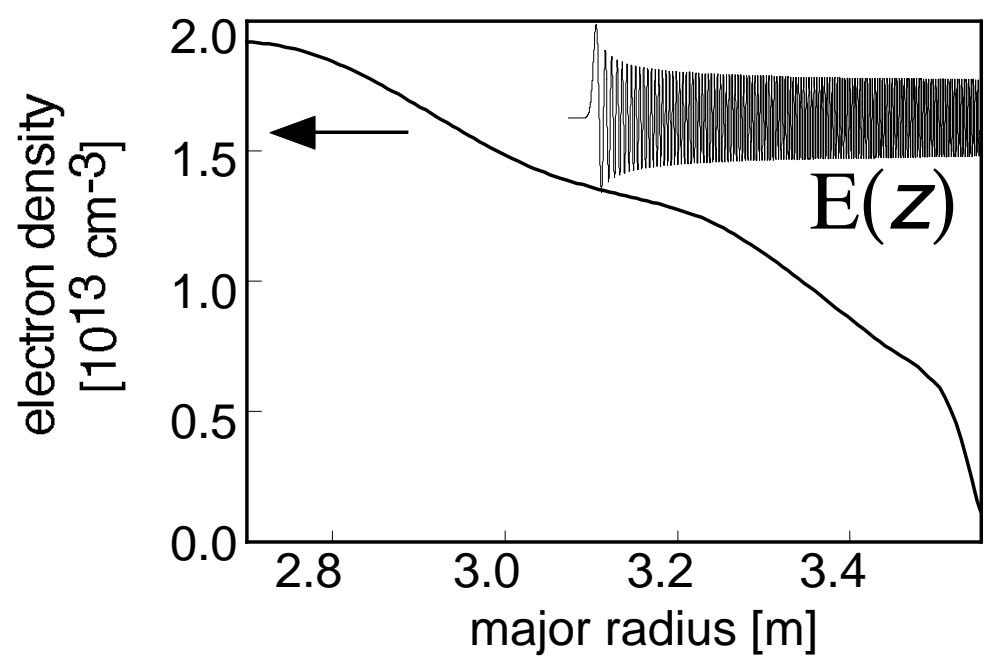

(a)

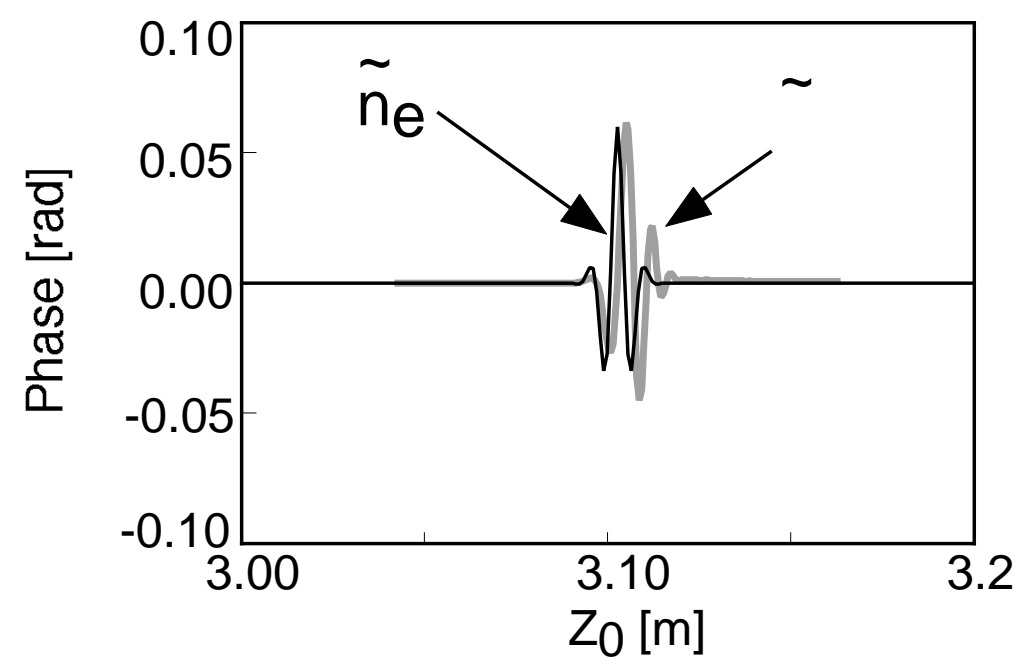

(b)

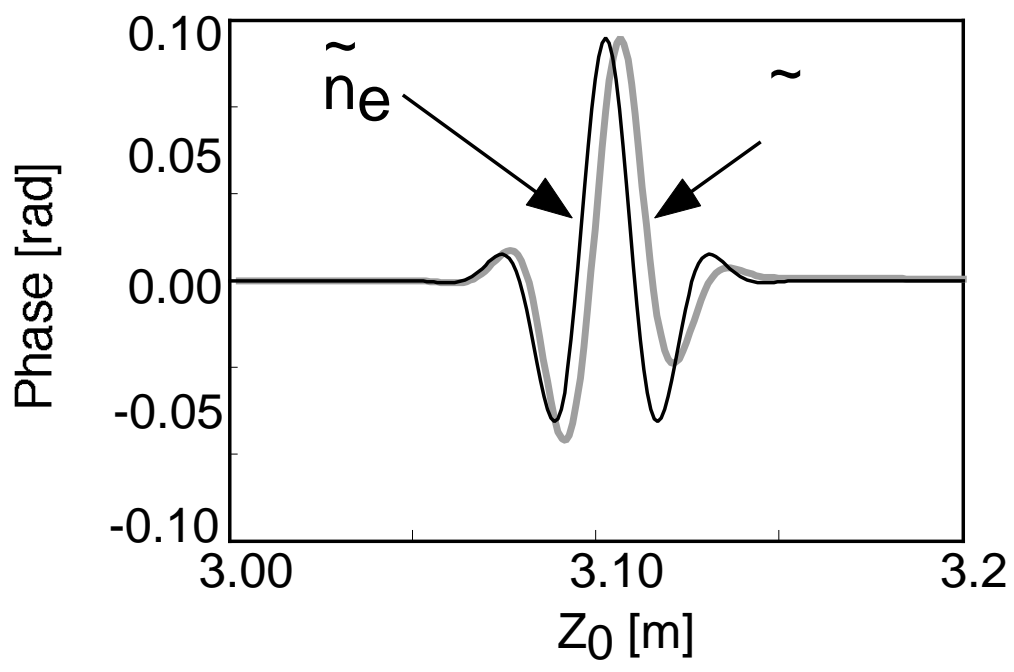

(c)

Figure.8

R. Nazikian 


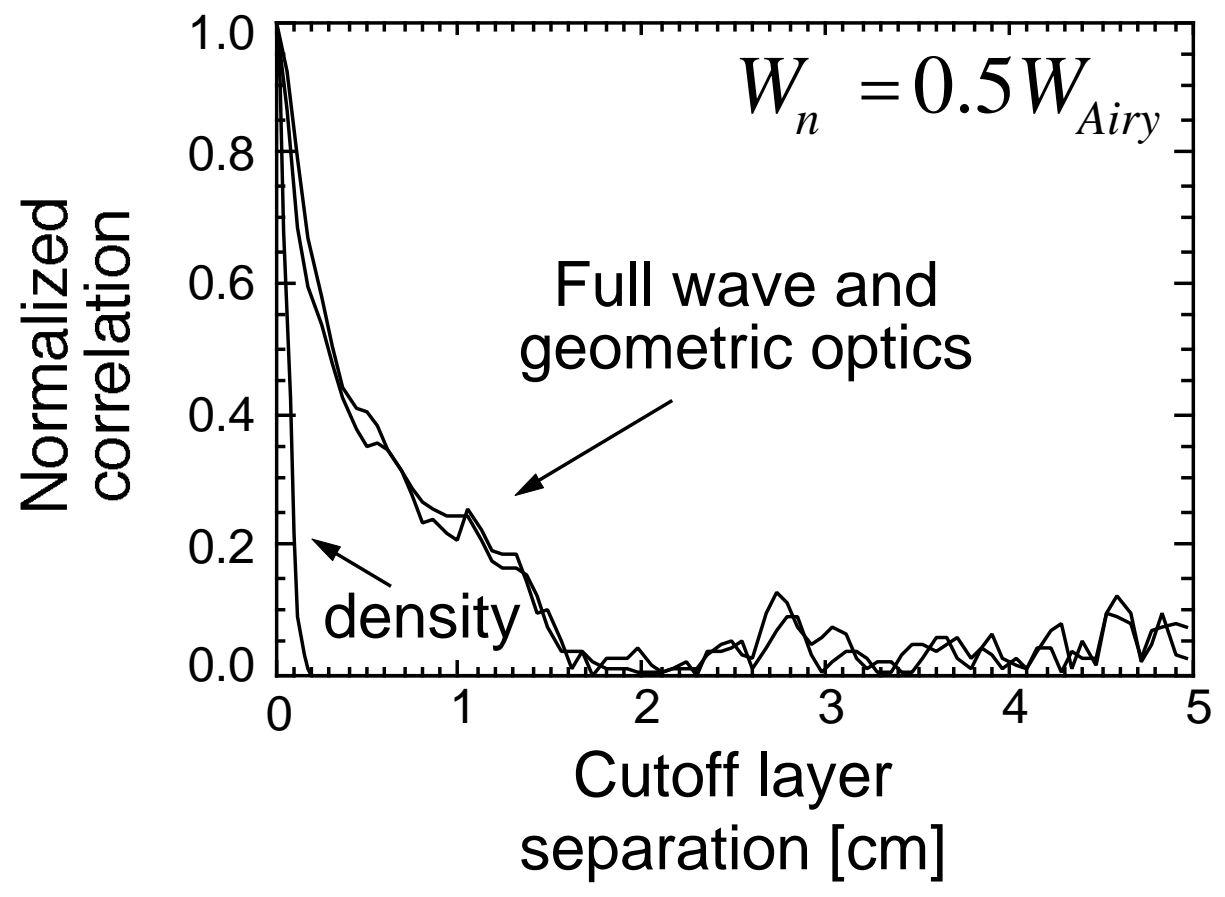

(a)

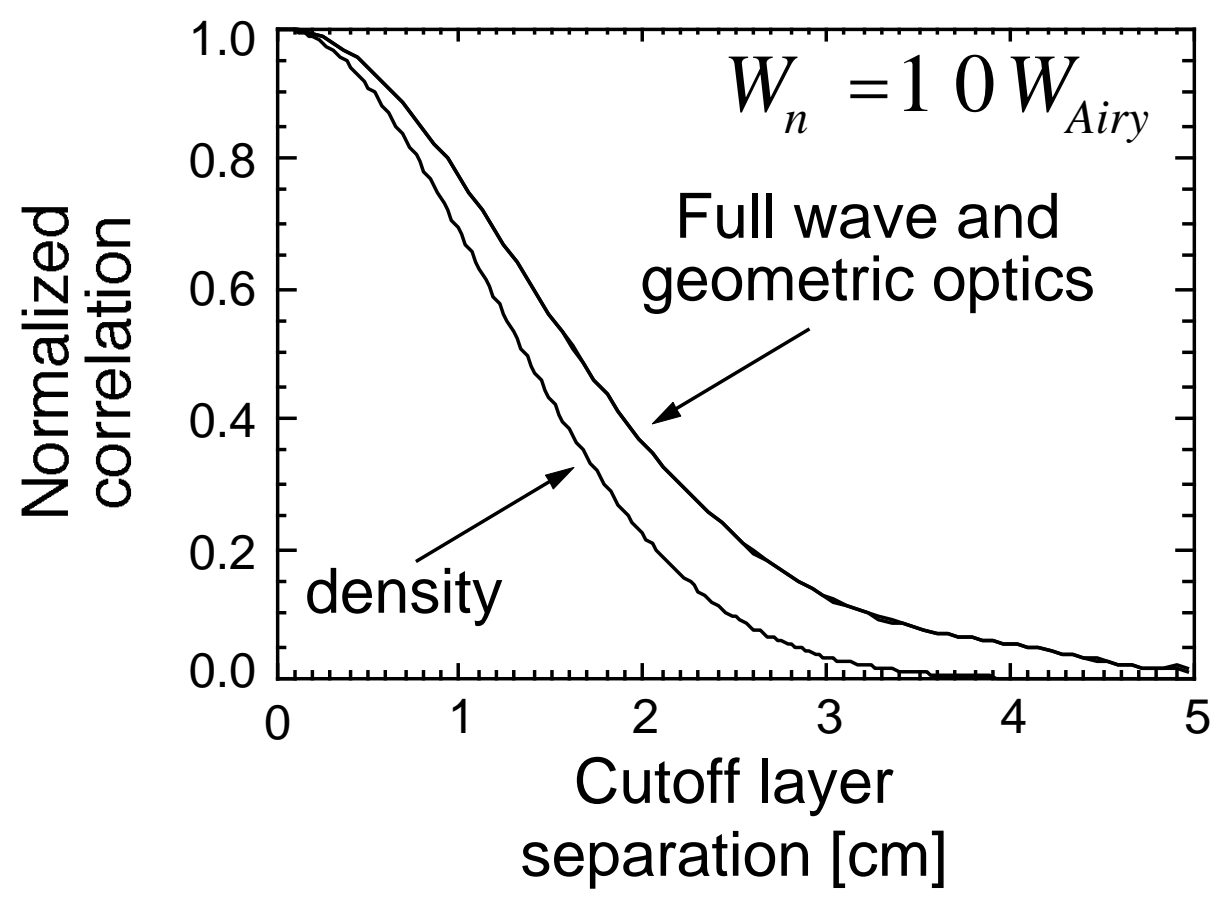

(b)

Figure 9

R. Nazikian 


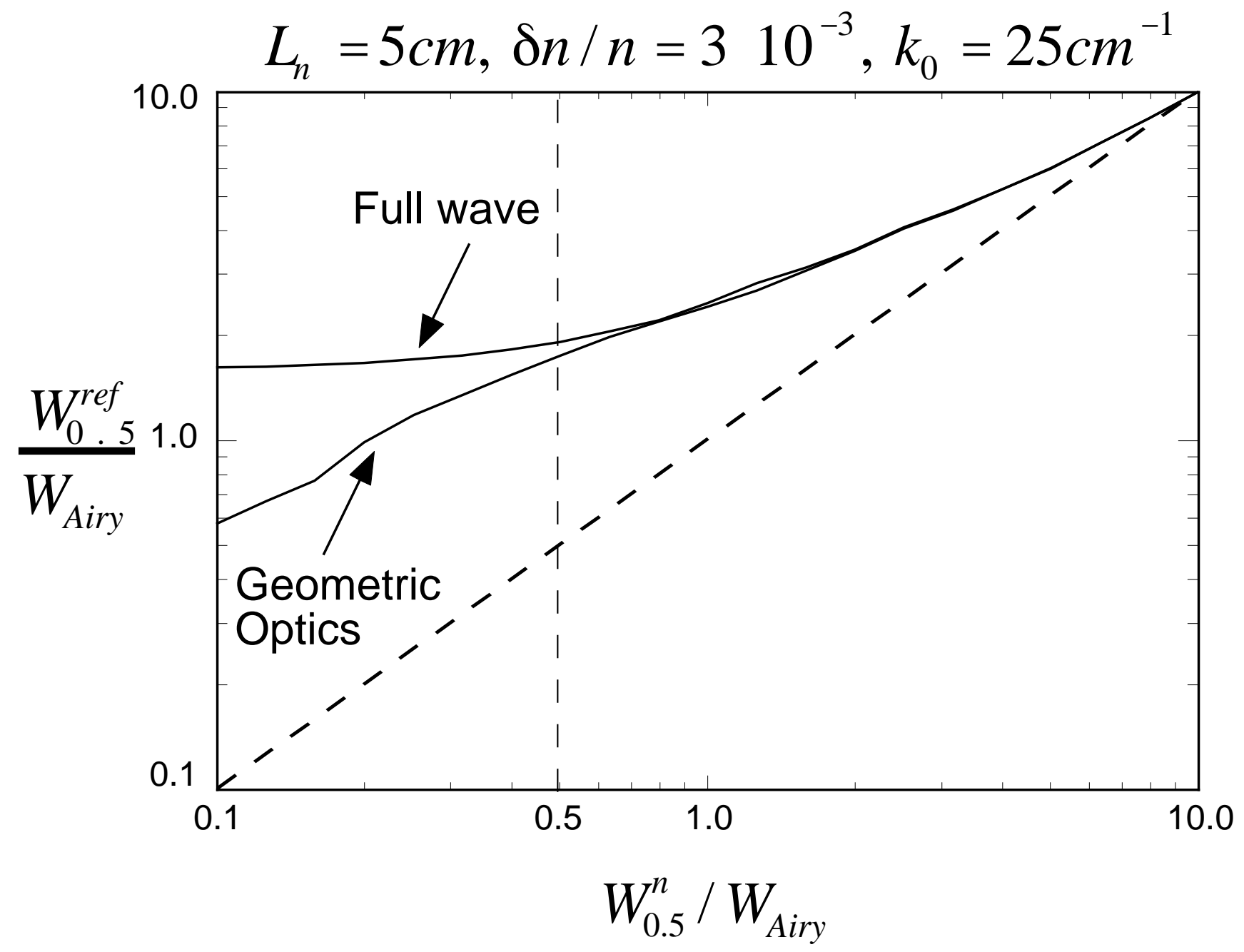

Figure 10 R. Nazikian 


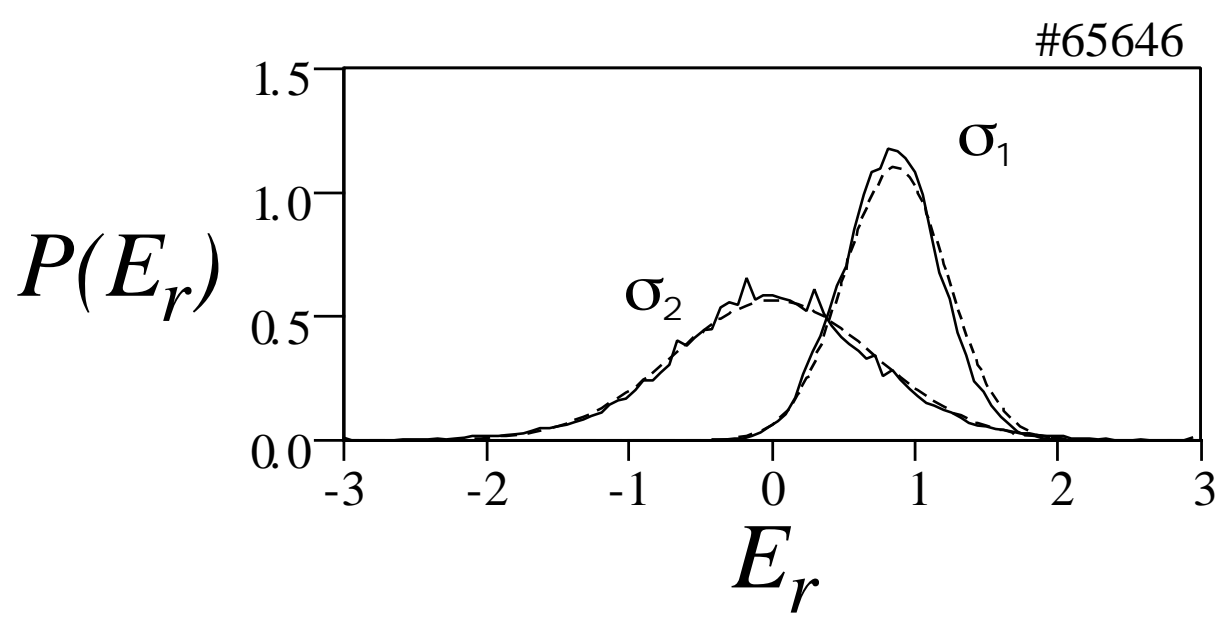

(a)

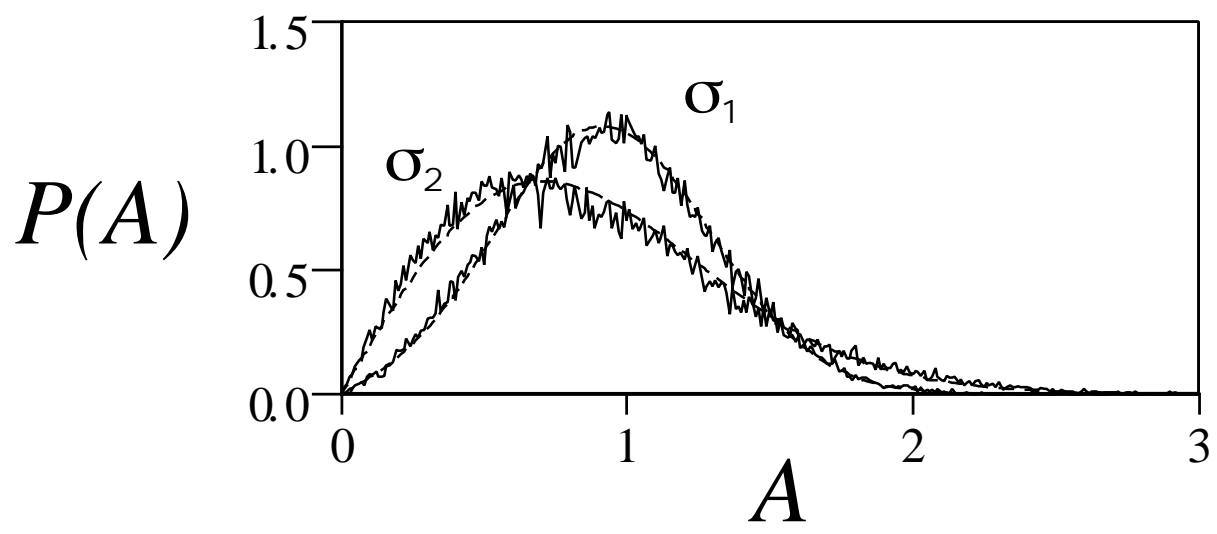

(b)

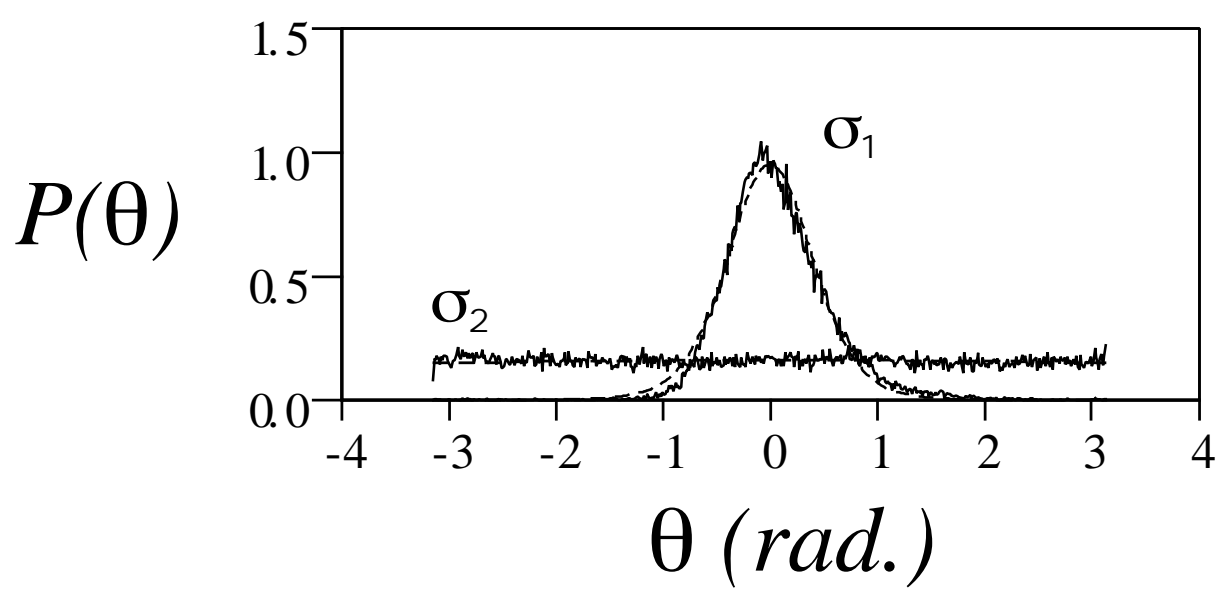

(c)

Figure 11

R. Nazikian 


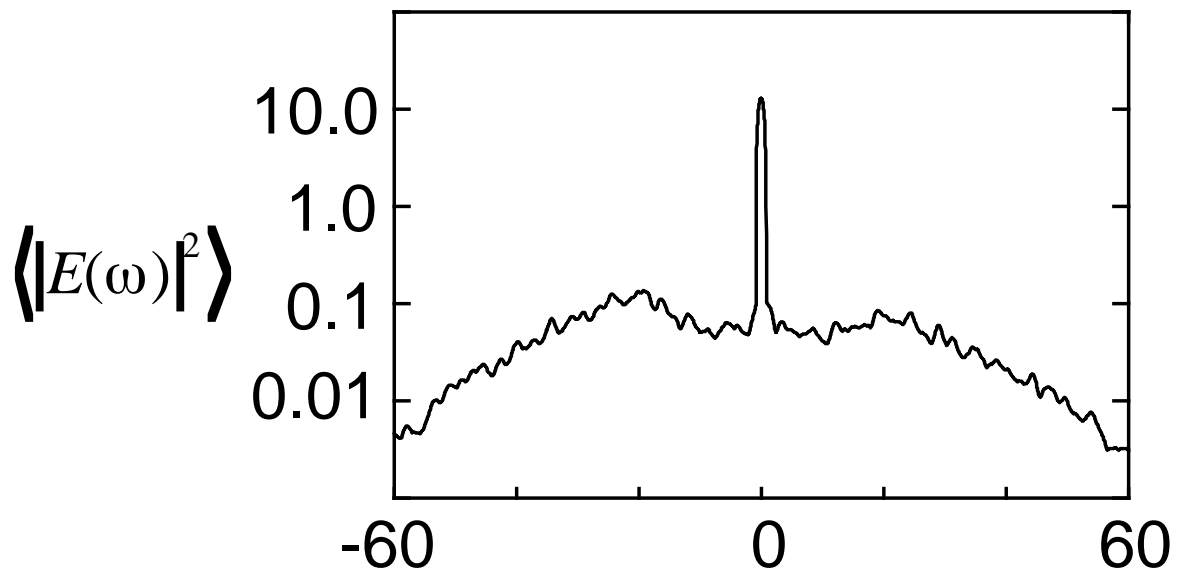

(a)

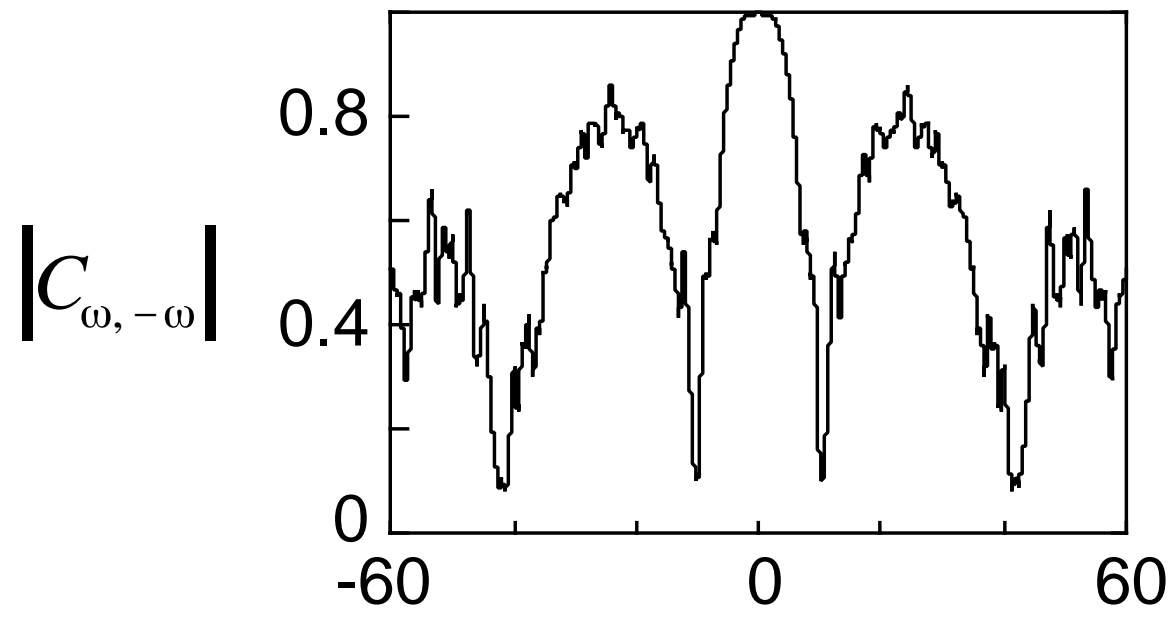

(b)

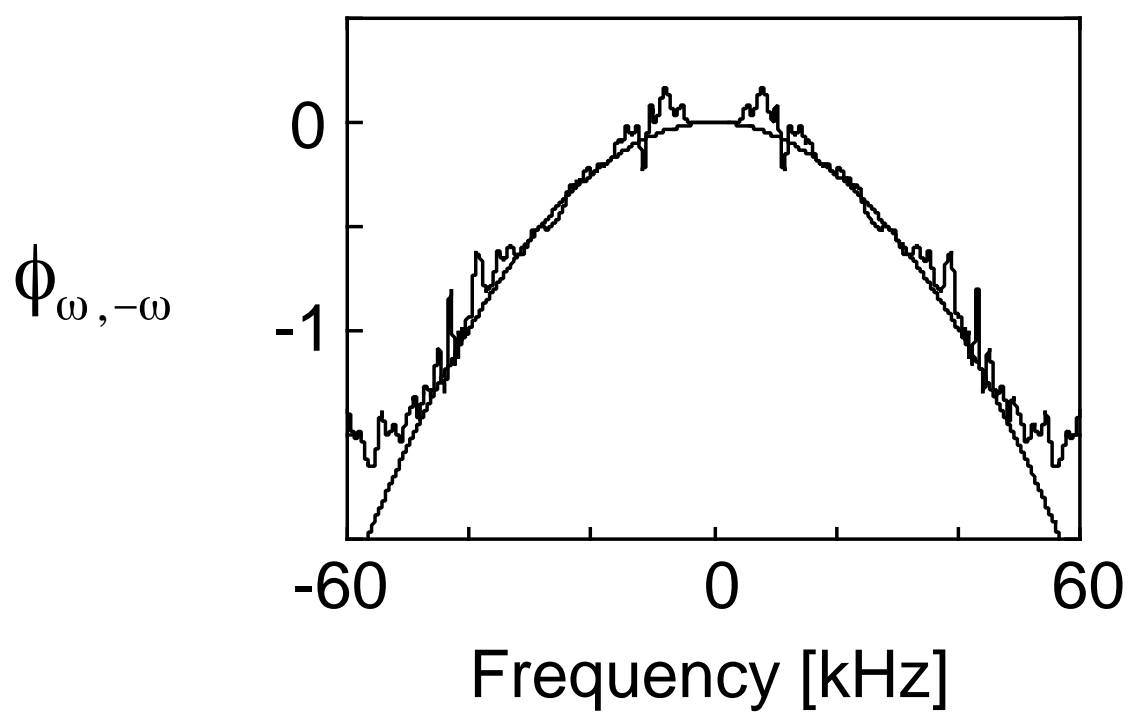

(c)

Figure 12

R. Nazikian 


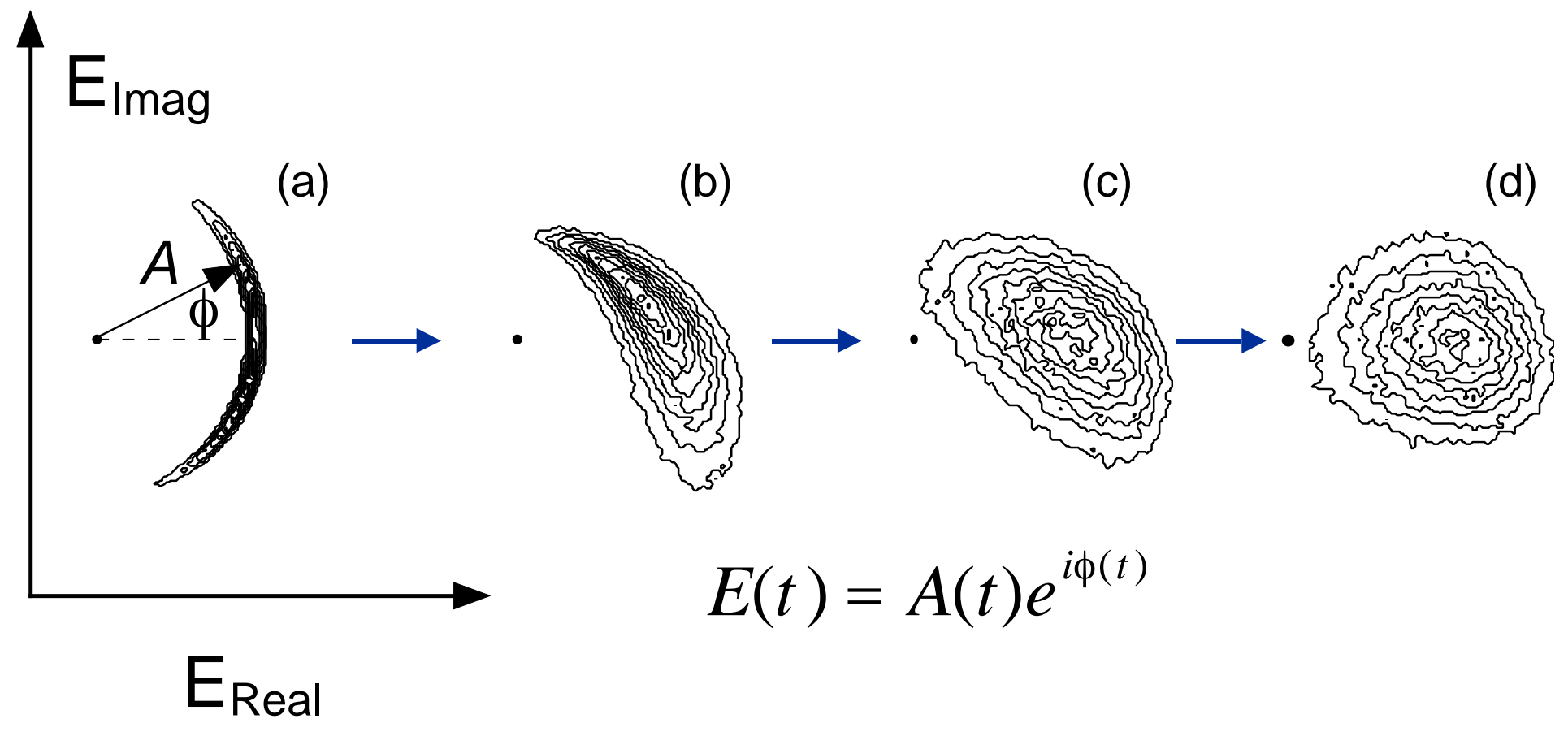

Figure.13

R. Nazikian 


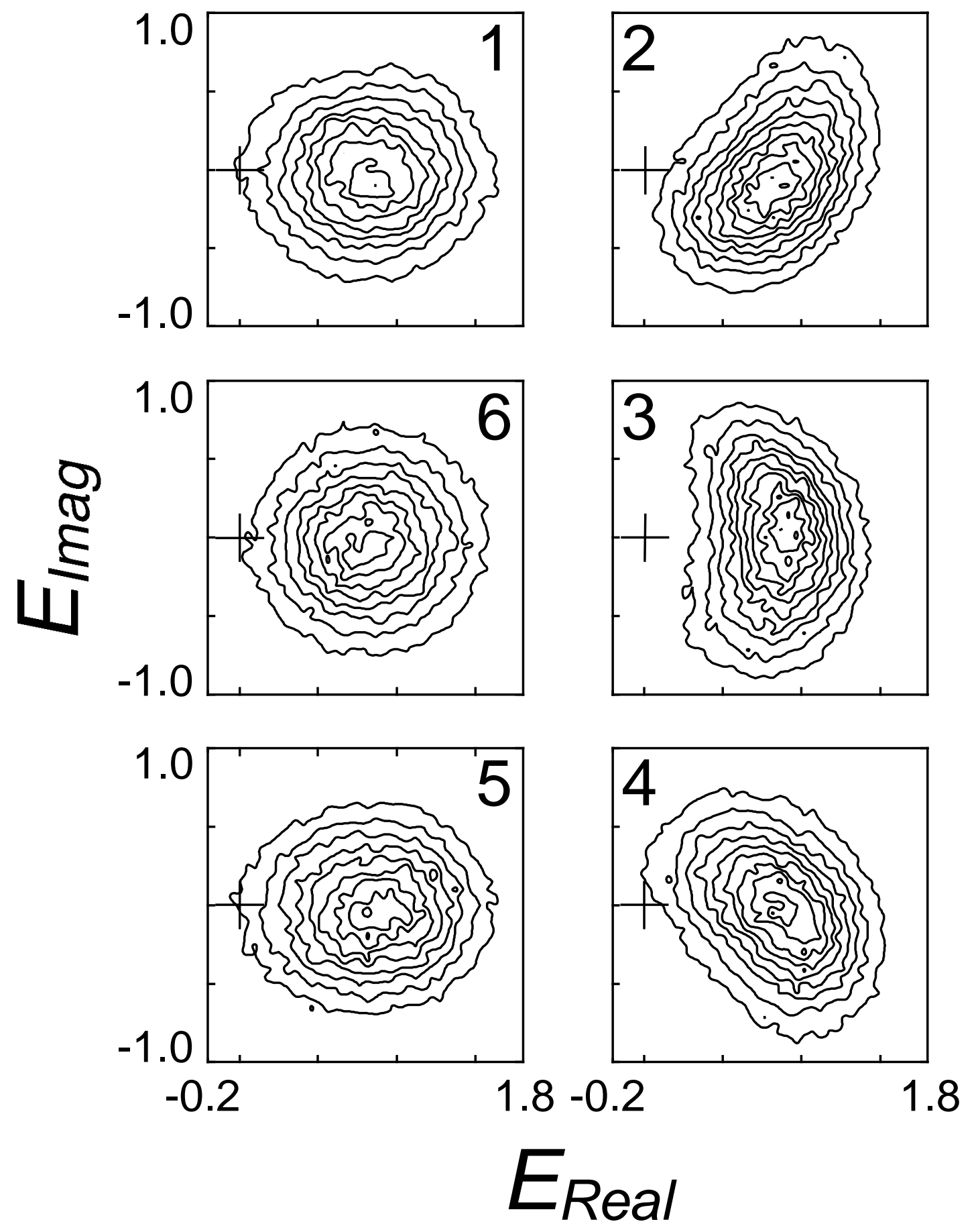

Figure.14

R. Nazikian 


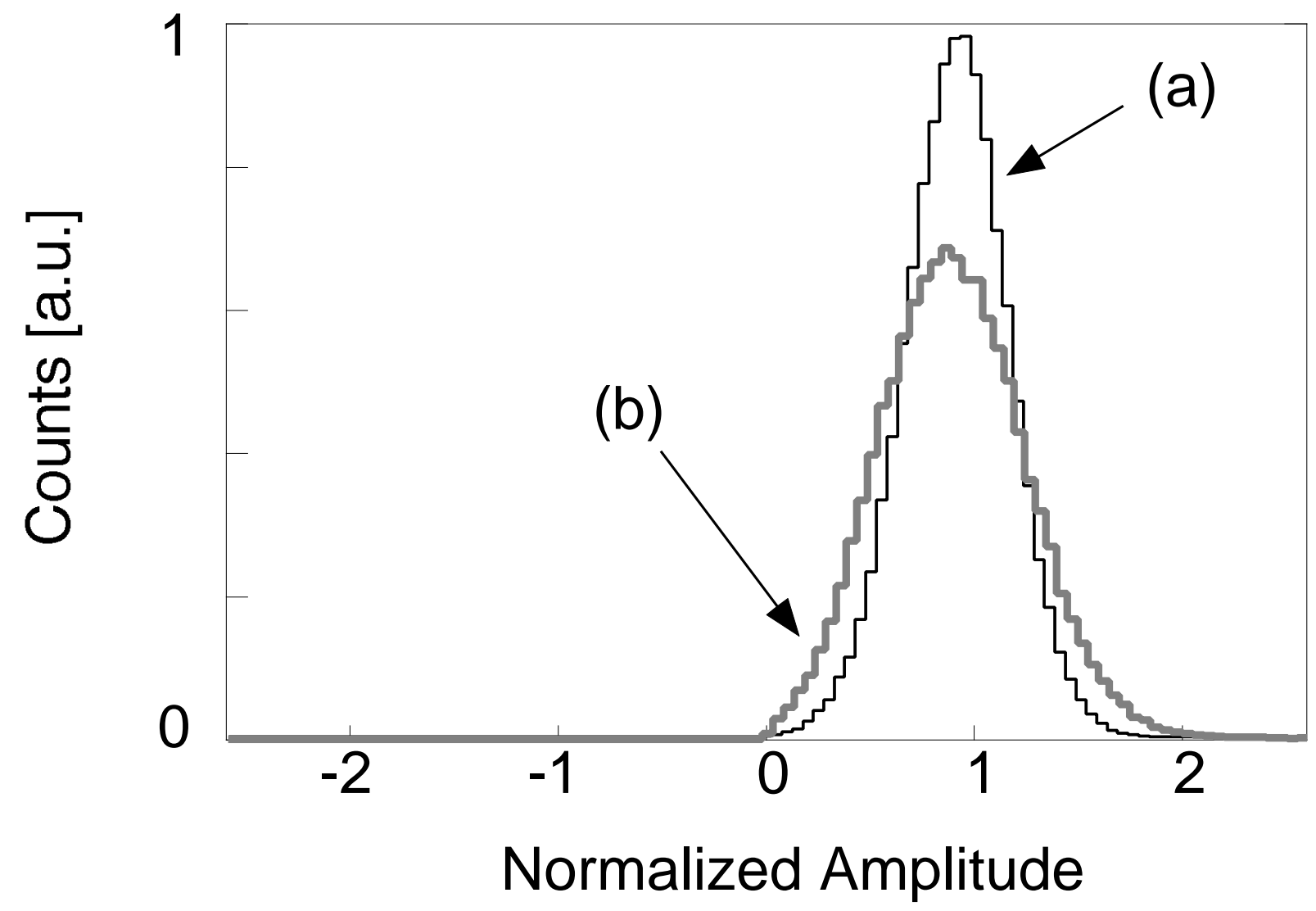

Figure 15

R. Nazikian 


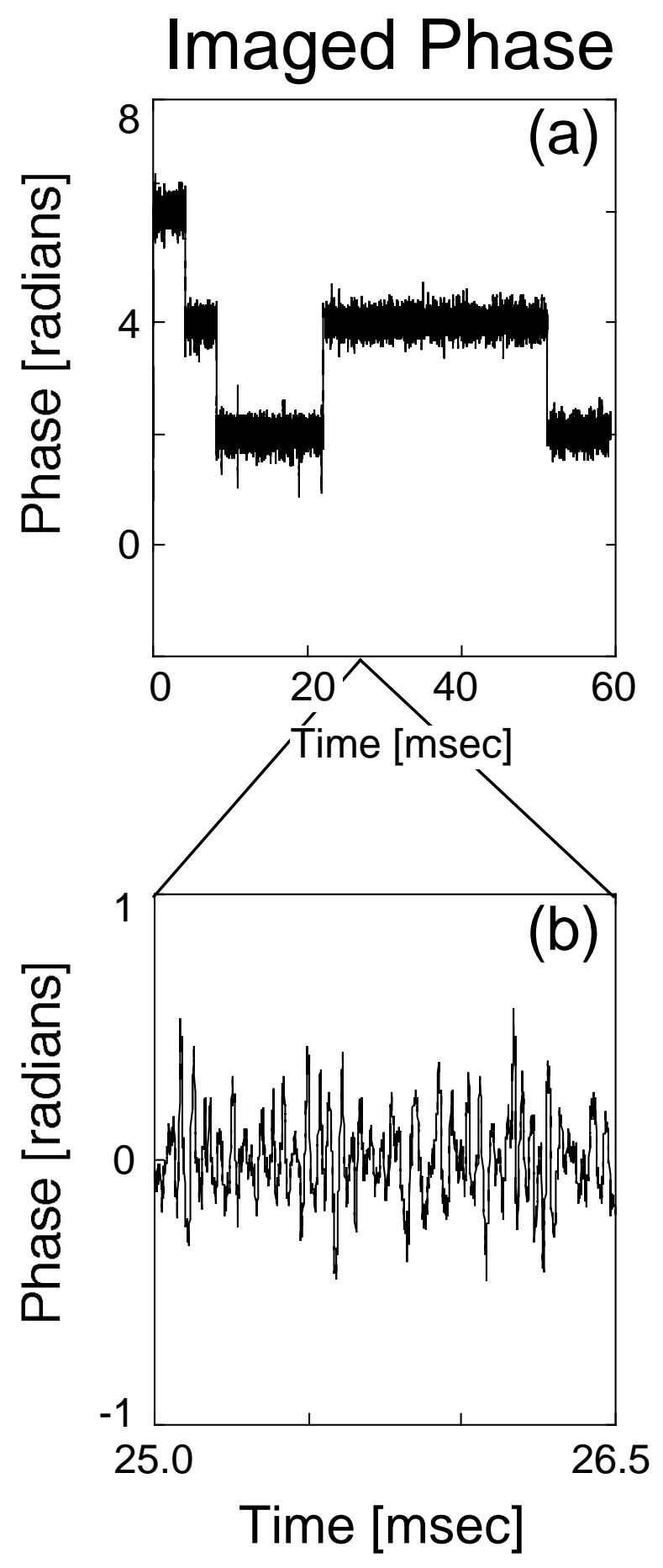

Far Field Phase
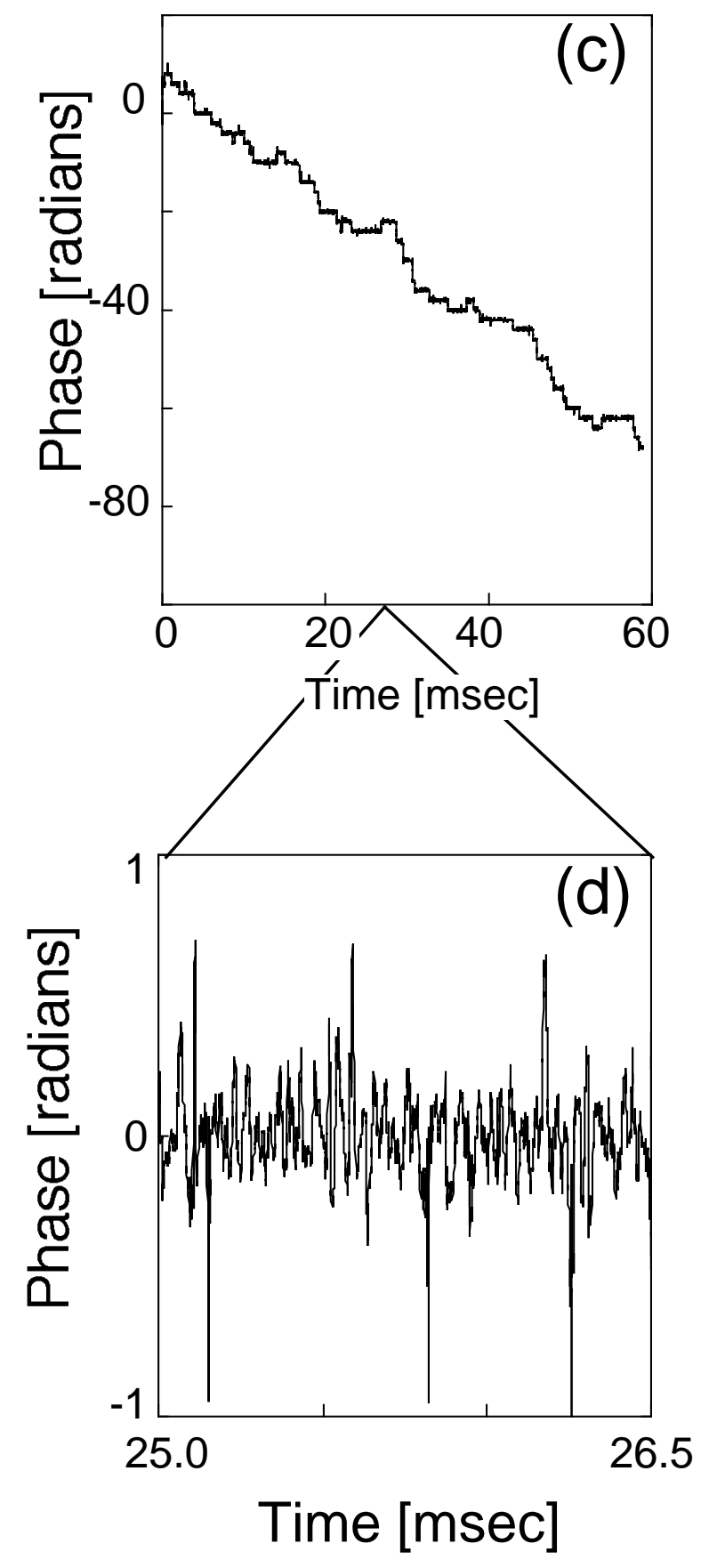

Figure 16

R. Nazikian 


\section{External Distribution}

Plasma Research Laboratory, Australian National University, Australia

Professor I.R. J ones, Flinders University, Australia

Professor J oão Canalle, Instituto de Fisica DEQ/IF - UERJ , Brazil

Mr. Gerson O. Ludwig, Instituto Nacional de Pesquisas, Brazil

Dr. P.H. Sakanaka, Instituto Fisica, Brazil

The Librarian, Culham Laboratory, England

Library, R61, Rutherford Appleton Laboratory, England

Mrs. S.A. Hutchinson, JET Library, England

Professor M.N. Bussac, Ecole Polytechnique, France

Librarian, Max-Planck-Institut für Plasmaphysik, Germany

J olan Moldvai, Reports Library, MTA KFKI-ATKI, Hungary

Dr. P. Kaw, Institute for Plasma Research, India

Ms. P.J . Pathak, Librarian, Insitute for Plasma Research, India

Ms. Clelia De Palo, Associazione EURATOM-ENEA, I taly

Dr. G. Grosso, Instituto di Fisica del Plasma, Italy

Librarian, Naka Fusion Research Establishment, J AERI, J apan

Library, Plasma Physics Laboratory, Kyoto University, J apan

Research Information Center, National Institute for Fusion Science, J apan

Dr. O. Mitarai, Kyushu Tokai University, J apan

Library, Academia Sinica, Institute of Plasma Physics, People's Republic of China

Shih-Tung Tsai, Institute of Physics, Chinese Academy of Sciences, People's Republic of China

Dr. S. Mirnov, Triniti, Troitsk, Russian Federation, Russia

Dr. V.S. Strelkov, Kurchatov Institute, Russian Federation, Russia

Professor Peter Lukac, Katedra Fyziky Plazmy MFF UK, Mlynska dolina F-2, Komenskeho Univerzita, SK-842 15 Bratislava, Slovakia

Dr. G.S. Lee, Korea Basic Science Institute, South Korea

Mr. Dennis Bruggink, Fusion Library, University of Wisconsin, USA

Institute for Plasma Research, University of Maryland, USA

Librarian, Fusion Energy Division, Oak Ridge National Laboratory, USA

Librarian, Institute of Fusion Studies, University of Texas, USA

Librarian, Magnetic Fusion Program, Lawrence Livermore National Laboratory, USA

Library, General Atomics, USA

Plasma Physics Group, Fusion Energy Research Program, University of California at San Diego, USA

Plasma Physics Library, Columbia University, USA

Alkesh Punjabi, Center for Fusion Research and Training, Hampton University, USA

Dr. W.M. Stacey, Fusion Research Center, Georgia Institute of Technology, USA

Dr. J ohn Willis, U.S. Department of Energy, Office of Fusion Energy Sciences, USA

Mr. Paul H. Wright, Indianapolis, Indiana, USA 
The Princeton Plasma Physics Laboratory is operated by Princeton University under contract with the U.S. Department of Energy.

\author{
Information Services \\ Princeton Plasma Physics Laboratory \\ P.O. Box 451 \\ Princeton, NJ 08543
}

Phone: 609-243-2750

Fax: 609-243-2751

e-mail: pppl_info@pppl.gov

Internet Address: http://www.pppl.gov 